\title{
Scaling of streamwise boundary layer streaks and their ability to reduce skin-friction drag
}

\author{
S. Shahinfar ${ }^{1}$, J. H. M. Fransson ${ }^{1, \dagger}$, S. S. Sattarzadeh ${ }^{1}$ and A. Talamelli ${ }^{1,2}$ \\ ${ }^{1}$ Linné Flow Centre, KTH Mechanics, SE-10044 Stockholm, Sweden \\ ${ }^{2}$ DIN, Alma Mater Studiorum - Università di Bologna, I-47100 Forlì, Italy \\ (Received 30 January 2013; revised 2 July 2013; accepted 12 August 2013; \\ first published online 19 September 2013)
}

Spanwise arrays of miniature vortex generators (MVGs) are used to generate energetic transient disturbance growth, which is able to modulate the boundary layer flow with steady and stable streak amplitudes up to $32 \%$ of the free-stream velocity. This type of modulation has previously been shown to act in a stabilizing manner on modal disturbance growth described by classical instability theory. In an attempt to reproduce a more realistic flow configuration, in the present experimental setup, Tollmien-Schlichting (TS) waves are generated upstream of the MVG array, allowing for a complete interaction of the incoming wave with the array. Fifteen new MVG configurations are investigated and the stabilizing effect on the TS waves is quantified. We show that the streak amplitude definition is very important when trying to relate it to the stabilization, since it may completely bypass information on the mean streamwise velocity gradient in the spanwise direction, which is an essential ingredient of the observed stabilization. Here, we use an integral-based streak amplitude definition along with a streak amplitude scaling relation based on empiricism, which takes the spanwise periodicity of the streaks into account. The results show that, applying the integral definition, the optimal streak amplitude for attenuating TS wave disturbance growth is around $30 \%$ of the free-stream velocity, which corresponds to $\sim 20 \%$ in the conventional definition when keeping the spanwise wavelength constant. The experiments also show that the disturbance energy level, based on the full velocity signal, is significantly reduced in the controlled case, and that the onset of transition may be inhibited altogether throughout the measured region in the presence of an MVG array.

Key words: drag reduction, instability control, transition to turbulence

\section{Introduction}

Over the past two decades, a large amount of research has been devoted to the study of transient disturbance growth in different wall-bounded shear layer flows. The pioneering work by Butler \& Farrell (1992) is seen as the springboard for the optimal 
perturbation theory, which for a long time was motivated by the worst-case disturbance scenario in the sense that it would be the far most dangerous disturbance, causing a bypass transition to turbulence at some downstream location.

Any transition route to turbulence that does not follow the classical modal disturbance description (see e.g. Tollmien 1929; Schlichting 1933; Schubauer \& Skramstad 1947) is classified as a bypass scenario (Morkovin 1969), since in that case the onset of a primary instability sets in at a subcritical Reynolds number with respect to modal theory and hence the disturbance growth described by the modal disturbance growth is completely bypassed. This is often the case when the background disturbance level exceeds a value of $\simeq 0.5 \%$ of the free-stream velocity, or if the wall-bounded flow develops on a surface with roughness (Kachanov 1994). In considering an optimization of the initial perturbation that maximizes the kinetic disturbance energy at a given time (or downstream distance), i.e. the optimal perturbation theory, the new non-modal approach has given rise to streamwiseorientated vortices that produce streaks of alternating high- and low-speed regions. These types of elongated structures had already been observed experimentally by Klebanoff (1971) in the free-stream turbulence-induced transition scenario.

In the linear framework, this disturbance growth is transient in terms of an algebraic disturbance growth followed by an exponential viscous decay for spatially invariant flows, or an algebraic viscous decay for spatially developing flows (Luchini 1996). The algebraic growth is a consequence of the non-normality of the governing differential operator. As the normal modes are not orthogonal, constructive and destructive interference may give rise to transients before the asymptotic state described by modal theory sets in (Schmid \& Henningson 2001). The transient growth and its linear physical mechanism was described by Ellingsen \& Palm (1975) and Landahl (1980), and since then a number of numerical works have been produced on the topic. Among the earlier ones are, for example, Hultgren \& Gustavsson (1981), Gustavsson (1991), Reddy \& Henningson (1993), Trefethen et al. (1993) and later also Andersson, Berggren \& Henningson (1999) Luchini (2000) and Reshotko (2001).

Some years ago, an appealing control strategy based on a passive mechanism was proposed by Fransson et al. (2006). By utilizing the transient disturbance growth as described above, they were able to attenuate the growth of classical modal disturbances and delay transition to turbulence. The positive aspect of a passive control method is, on the one hand, the idea that no energy has to be added to the system in order to perform the control. On the other hand, nor does the method have to rely on any sensitive electronics in a fancy sensor-actuator control system, which makes the control fairly robust when compared with alternative methods.

In their study, they experimentally generated streamwise streaks by using circular roughness elements mounted in an array on the surface perpendicular to the main stream (Fransson et al. 2004), which had been shown to be an effective configuration in damping the growth of Tollmien-Schlichting (TS) waves (Fransson et al. 2005), and they were able to deliver a first proof of the concept of the passive control strategy for transition delay (Fransson et al. 2006). These experimental results have recently been confirmed by large-eddy simulations (Schlatter et al. 2010). The stabilizing mechanism had previously been reported by Cossu \& Brandt (2002) and described with linear stability theory by Cossu \& Brandt (2004), as being associated with the extra turbulence production term arising when the boundary layer is modulated in the spanwise direction. The spanwise production term, with the covariance of the streamwise and spanwise velocity fluctuations acting on the mean streamwise velocity gradient with respect to the spanwise direction, was shown to be of negative sign. 
This quantity, together with the viscous dissipation, can overtake the positive wallnormal production term, always present in boundary layer flows and, overall, act in a stabilizing manner. Even though not fully understood, this stabilizing effect has already been observed experimentally and reported in the past by Kachanov \& Tararykin (1987) in the presence of steady streamwise streaks and Boiko et al. (1994) in the presence of unsteady streaks. However, in their studies they concluded that, in fact, as soon as the flow becomes nonlinear a streaky base flow promotes transition. A numerical study on unsteady streaks and the interaction with TS waves was reported by Liu, Zaki \& Durbin (2008), confirming the experimental result that unsteady streaks promote transition. In addition to these works, unsteady streaks have, on the other hand, been shown to damp the evolution of turbulent spots in spatially invariant boundary layers (Fransson 2010), indicating that the stabilization may also work in the turbulent regime.

For a successful experimental stabilization of the flow, which also works in the nonlinear regime and for a significant transition delay, the streaky base flow has to be set up with great care, paying attention to making sure that it is 'stable', which is the major challenge of this control method. Different ways to proceed experimentally in generating streamwise streaks have been reported in the past, most studies having been related to pure transient growth studies (see e.g. White 2002; Kurian \& Fransson 2011) and secondary instability studies (see e.g. Asai, Minagawa \& Nishioka 2002). We refer to Fransson \& Talamelli (2012) for a detailed review of this subject.

In Fransson \& Talamelli $(2011,2012)$, a second step towards real flow applications was taken within the AFRODITE programme (Fransson et al. 2011) by replacing the array of circular roughness elements by an array of miniature vortex generators (MVGs). These devices are miniature with respect to the classical vortex generators typically used for separation delay in both laminar and turbulent boundary layers. Fransson \& Talamelli $(2011,2012)$ were able to show that streak amplitudes of up to $32 \%$ of the free-stream velocity can be generated using MVGs. Beyond this threshold amplitude, the streaks become unstable and transition to turbulence through a secondary instability mechanism at some distance downstream of the array. The transition mechanism in the streaks produced by MVGs is believed to be different from those generated by circular roughness elements. In the latter, the onset of transition is local, at the position of the array, with the main instability originating from the wake behind the roughness elements, similarly to a vortex-shedding type behind bluff bodies. Conversely, an MVG pair consists of two small blades, positioned at a certain angle of attack, which allows the flow to pass straight through them, reducing the size of the wake compared to a solid bluff body. The stability of the streaks produced by the two different methods can be quantified by comparing their corresponding critical Reynolds numbers, based on the height of the devices. Fransson \& Talamelli (2012) reported a critical blade height Reynolds number of 547, compared to the corresponding roughness height Reynolds number of the circular elements of 420 (cf. Fransson et al. 2005). It should be pointed out that the streak amplitude depends on many parameters, such as the blade angle against the centre line, $\theta$, the geometry of the blades (triangular versus rectangular), the length of the blades, $L$, and consequently also the critical height Reynolds number. Therefore, conclusions arrived at by means of a direct comparison of this number between different geometrical devices should be treated with caution. However, for a fixed angle (e.g. $\theta=15^{\circ}$ ), an equivalent diameter of the circular roughness element may be used as the distance between the blades, which then gives the same streak amplitude. However, it is not only the streak amplitude that determines the effectiveness in damping incoming TS 
waves, but also the devices. Recent results by Shahinfar et al. (2012) have shown that MVGs are much more effective than circular roughness elements in damping incoming TS waves and delay transition in a more realistic flow configuration. In this study, the TS waves were generated upstream of the physical devices mounted in an array on a flat plate. This is a more realistic configuration, since the complicate interactions between the incoming disturbances and the MVG array are fully taken into account - as opposed to Fransson et al. (2006) and later Schlatter et al. (2010), where the TS waves were generated in a streaky base flow that had already developed.

In the present paper, we report a thorough parametric study on the stabilization of TS waves using the above-described passive flow control strategy by testing different MVG configurations. In previous works, (including in Fransson \& Talamelli 2012), the streak amplitude definition was based on the maximum velocity difference $(\Delta U)$ measured on the horizontal line above the wall corresponding to the maximum $\Delta U$ in the wall-normal direction. This definition, often as a percentage of the free-stream velocity, was based solely on two single-point measurements, even though a full crosssectional scan had to be made in identifying the location above the wall of maximum $\Delta U$. Considering a numerical simulation, this definition can be quite accurate, due to the high grid resolution and the presence of perfectly periodic streaks. In experiments, where lower grid resolutions are typically used, this definition could under-predict the true streak amplitude. Moreover, it is inappropriate when trying to relate it to the stabilization of disturbances, since it completely bypasses information on the gradient in the spanwise direction of the mean streamwise velocity component, which is an essential ingredient of the observed stabilization. An integral definition applied to the variance of the velocity difference has been used by White (2002) and White, Rice \& Ergin (2005). In this way, it was possible to improve the accuracy of the streak measure, be it energy or amplitude, and to take the velocity variations over the entire cross-sectional plane into account. Here, we propose a similar integral definition, which takes the spanwise periodicity of the streaks into account, but is still defined as a percentage of the free-stream velocity. This definition allows us to find a scaling relation of the streaky base flow based on empiricism. As a result, we are able to show the attenuation of the incoming TS wave as a function of the streak amplitude and that the MVGs are, in this particular configuration, effective devices in obtaining transition delay and hence skin-friction drag reduction.

\section{The experimental set-up}

The experiments were performed in the minimum turbulence level (MTL) wind tunnel at the Royal Institute of Technology (KTH) in Stockholm, Sweden. The MTL is a closed circuit tunnel with a test section of $7 \mathrm{~m}$ in length and a cross-sectional area of $0.8 \mathrm{~m}$ and $1.2 \mathrm{~m}$ in height and width, respectively. The flow is provided by an axial fan (DC $85 \mathrm{~kW}$ ), which can be operated in the range $\simeq 0.5-70 \mathrm{~m} \mathrm{~s}^{-1}$ in an empty test section. The flow quality in this tunnel is considered to be good, with a background turbulence level of $0.025 \%$ of the free-stream velocity at $25 \mathrm{~m} \mathrm{~s}^{-1}$ (Lindgren \& Johansson 2002). Downstream of the fan a heat exchanger is installed, which is capable of maintaining the circulating air at a constant temperature with a tolerance of $\pm 0.05{ }^{\circ} \mathrm{C}$ above $6 \mathrm{~m} \mathrm{~s}^{-1}$. The boundary layer experiments were performed on a $4.2 \mathrm{~m}$ long flat plate positioned horizontally inside the test section. An asymmetric leading edge was used (Klingmann et al. 1993) along with an adjustable trailing edge flap to minimize, by reducing the pressure gradient region, the leading edge effect. A sketch of the plate inside the MTL is shown in figure 1, where the tunnel coordinates are 




(b)

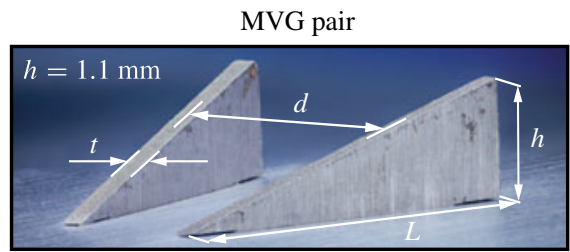

FIGURE 1. (Colour online) (a) A schematic of the flat plate with the evolving boundary layer. Region (I) corresponds to the laminar boundary layer. The black and white stripy pattern perpendicular to the main stream downstream of the disturbance slot in region (II) indicates the TS waves. In region (III), a similar stripy pattern aligned with the main stream indicates the alternating high- and low-speed streaks. In region (IV), the two-dimensional laminar flow is recovered. (b) A photograph of an MVG pair with corresponding geometrical parameters (see table 1).

defined with $(x, y, z)$ as the streamwise, wall-normal and spanwise coordinates. The corresponding velocity components are $(U, V, W)$, with small letters denoting velocity perturbations. The averaged value of an arbitrary quantity, $q$, in the spanwise direction (z) or in time $(t)$, is denoted with a superscript as $q^{z}$ or $q^{t}$, respectively. To measure the velocity signal, a constant-temperature hot-wire anemometer was employed. In all the measurements, a DANTEC Dynamics ${ }^{\mathrm{TM}}$ StreamLine $90 \mathrm{~N} 10$ anemometer system was used and the voltage signals were acquired by a National Instruments ${ }^{\mathrm{TM}}$ acquisition board (NI PCI-6259, 16-Bit). Single-wire probes were manufactured in-house, using Wollaston platinum wires of $2.54 \mu \mathrm{m}$ in diameter and $0.7 \mathrm{~mm}$ long. The hot wires were calibrated in-situ versus a Prandtl tube connected to a manometer (Furness FC0510). In order to get the best accuracy, especially close to the surface plate where the speed is low, a modified King's law was used as calibration function (Johansson \& Alfredsson 1982).

\subsection{Generation of streamwise streaks}

In order to generate streamwise vortices inside the boundary layer, a row of triangularbladed miniature vortex generators (MVGs) similar to those used by Fransson \& Talamelli (2012) was designed, manufactured and positioned by means of a flushed insert on the plate at $x_{M V G}=222 \mathrm{~mm}$ from the leading edge (figure 1). The blades were kept at the constant angle of attack of $\theta=15^{\circ}$ to the free-stream direction. The blade length $(L)$ and thickness $(t)$ were 3.25 and $0.3 \mathrm{~mm}$, respectively. The height of the MVGs $(h)$, the distance between two blades constituting an MVG pair $(d)$ and the spanwise distance between MVG pairs $(\Lambda)$ were here varied in order to obtain different MVG configurations and in turn different streaky boundary layers.

\subsection{Generation of Tollmien-Schlichting waves}

Tollmien-Schlichting (TS) waves were generated by means of six loudspeakers, which were tightly sealed and connected with 40 tubes to a slot in a plug flush mounted in the flat plate (see figure 1). In feeding a sinusoidal voltage signal with a prescribed 
forcing frequency from a computer, via amplifiers, to the loudspeakers, a continuous blowing and suction disturbance is being introduced at the surface, which sets up the TS wave inside the boundary layer. The measurements are triggered based on a selected phase of the sinusoidal output voltage from the computer. Three hifi amplifiers were used for the six loudspeakers in order to easily calibrate the initial forcing amplitude of the loudspeakers, to obtain a homogeneous spanwise distribution of the blowing and suction at the surface. The TS wave slot is located at $x_{T S}=190 \mathrm{~mm}$ from the leading edge. Throughout this paper, we use the nondimensional frequency $F=\left(2 \pi f_{0} \nu / U_{\infty}^{2}\right) \times 10^{6}$, where $f_{0} \mathrm{~Hz}$ is the forcing frequency and $v$ is the kinematic viscosity calculated using Sutherland's law and the ideal gas law, using the local conditions during the actual experiment.

\subsection{The measured configurations}

In this investigation, we consider different MVG configurations obtained by combining the following parameters: $h=1.1,1.3,1.5 \mathrm{~mm}, d=3.5,16.25 \mathrm{~mm}$ and $\Lambda=13,19.5,26 \mathrm{~mm}$. All these configurations are summarized in table 1, in which case $\mathrm{C} 01$ is denoted as the base configuration. With this geometrical configuration, five cases have been generated (C01, C07, $\mathrm{C} 08, \mathrm{C} 10$ and $\mathrm{C} 12)$, where $U_{\infty}, F$ or the initial forcing amplitude of the TS wave has been varied. The C04 configuration is similar to the base configuration, but with one blade removed in each pair. In this way, the MVGs are supposed to generate directly an array of co-rotating vortices.

The measurements of the streaky boundary layers were performed in nine $y z$ planes in the downstream direction, with a total number of 20 measurement points in the wallnormal direction. The distribution in $y$ increased with a power of 1.6 in order to have a higher resolution near the wall with the same number of points. The first measurement point, closest to the wall, was set to $0.4 \mathrm{~mm}$ above the wall in all measurements. The corresponding dimensionless distance above the wall $\eta=y / \delta$, where $\delta=\sqrt{\nu x / U_{\infty}}$, is in the range $0.60-0.32$ with increasing downstream distance for the C01 configuration. The last measurement point in the free stream was consistently set to $\eta=10$. In the spanwise direction, the probe was traversed one spanwise wavelength, i.e. in the range $\zeta=z / \Lambda=-0.5: 0.5$, with a step of $1 \mathrm{~mm}$ independent of $\Lambda$ and the downstream location. In outer units, the spanwise traverse is in the range $z / \delta=19.4-10.3$ with increasing downstream location for the C01 configuration. The locations in $x$ were chosen on the basis of the location of branch II of the neutral stability curve of the reference two-dimensional base flow, i.e. they were a function of $U_{\infty}$ and $f_{0}$. Each point was measured with four sets of $3 \mathrm{~s}$ per set and with a sampling frequency of $2 \mathrm{kHz}$. For configuration C01, each set corresponds to a sampling time of 265 time periods of the TS wave. The total measurement times, including probe traversing, were $\sim 11$ and $21 \mathrm{~h}$ for $\Lambda=13$ and $26 \mathrm{~mm}$, respectively.

\section{The reference base flow in the absence of MVGs}

In figure 2 the reference base flow, i.e. without the MVGs mounted on the surface, with a free-stream velocity of $\sim 6 \mathrm{~m} \mathrm{~s}^{-1}$ is shown. This boundary layer flow, at different free-stream velocities, will provide the reference cases to compare with, when the different MVG configurations and their effect on the growth of TS waves in $\S 5.1$ and on the transition delay in $\$ 5.2$ are presented.

Figure 2(a) shows the pressure distribution along the plate in terms of the pressure coefficient, $c_{p}=1-\left(U_{\infty}(x) / U_{r e f}\right)^{2}$, where $U_{r e f}$ is an adequate reference velocity close to the streamwise averaged free-stream velocity. The error bars correspond 
Streamwise streaks for skin-friction drag reduction

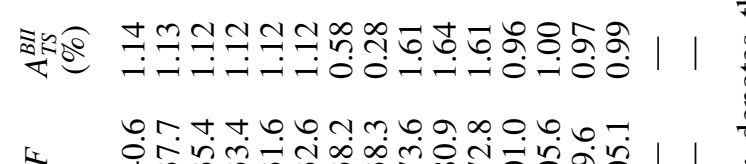

$\checkmark \Phi)$

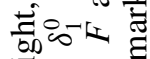

ब्త

$\checkmark$

$>000$

$\sum \sum_{i}$

뜨를

\%

苂. Е

ष्ठ

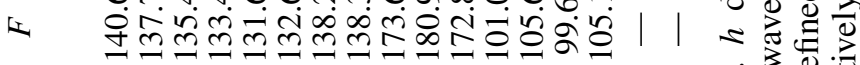

$\dot{s} \theta$



\& \&

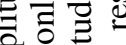

:을

苟

ฮี ํํㅇ

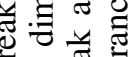

Q

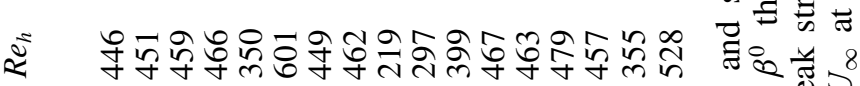

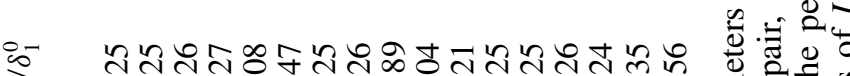



$8^{\text {In }}$ n.

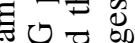

灵灵

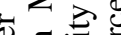

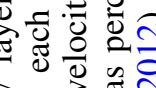



$\exists . \Xi$

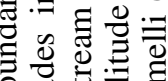



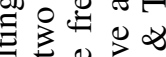

๕ 000000000000 . $0.00 \infty$

ব 西



$=\underset{\Xi}{\pi}$

बृ

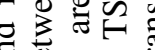



워효

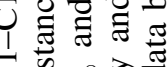

竞 $\square * 0 \triangleleft \Delta \nabla \Delta \diamond \mathbf{D} \bullet \bullet \nabla \Delta \bullet \oplus \otimes$ 응 8 它要

$\stackrel{0}{0}$

ڤ



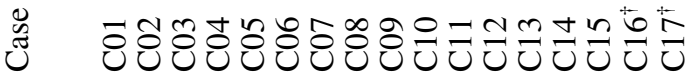



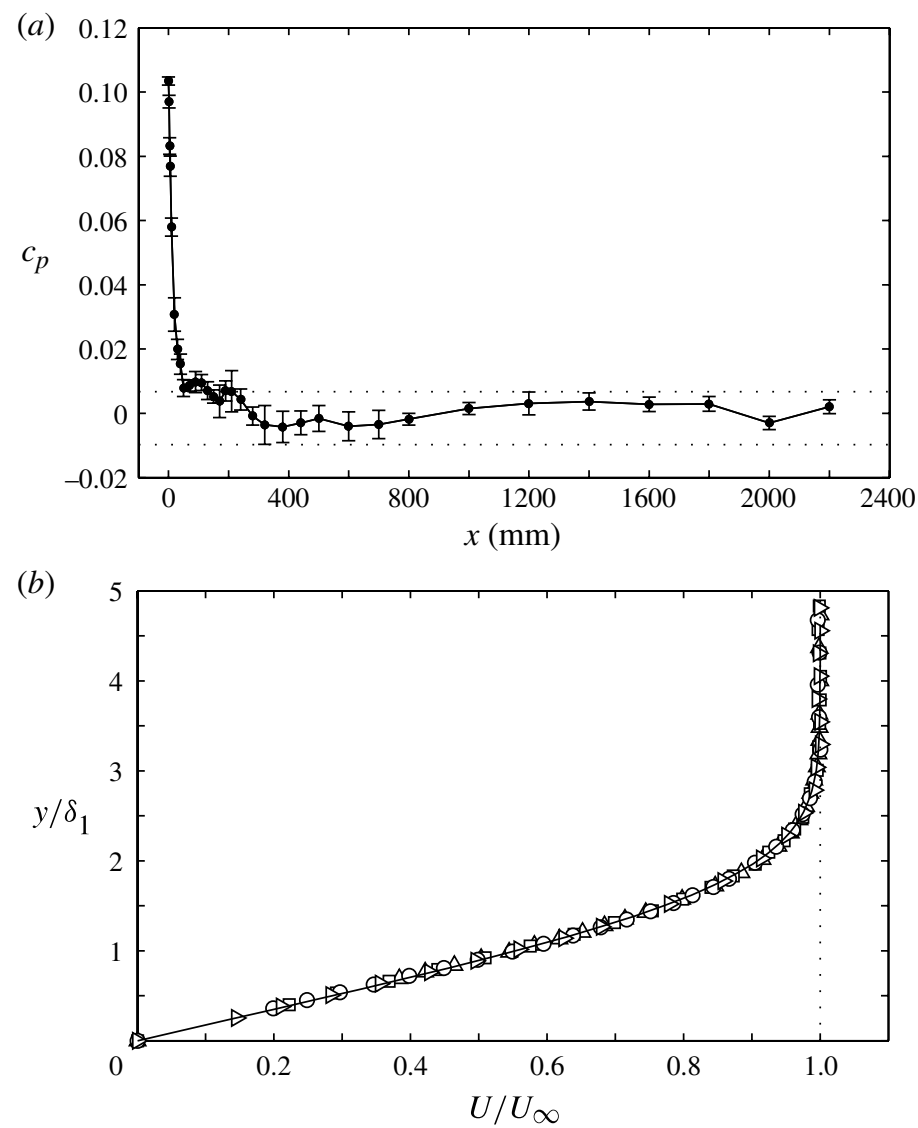

FIGURE 2. (a) The pressure coefficient distribution along the streamwise direction. The dashed lines show the range of pressure variation after the initial leading edge drop $\left(\Delta c_{p}=0.016\right)$. (b) Streamwise velocity profiles measured at different downstream locations $(\triangle, x=250 \mathrm{~mm} ; \square, x=500 \mathrm{~mm} ; O, x=1000 \mathrm{~mm} ; \triangleright, x=2000 \mathrm{~mm})$. The solid line corresponds to the Blasius solution.

to the standard deviation values, which have been calculated on the basis of five independent measurements, each one of $10 \mathrm{~s}$ sampling time. Figure $2(b)$ shows the mean streamwise velocity profiles in the wall-normal direction at four different streamwise positions, namely $x=(250,500,1000,2000) \mathrm{mm}$. The averaged values of the displacement thickness, $\delta_{1}$, and the momentum thickness, $\delta_{2}$, along with the shape factor, $H_{12}$, plus or minus half their standard deviations of the plotted profiles, become $\delta_{1} / \delta=1.65 \pm 0.02, \delta_{2} / \delta=0.62 \pm 0.01$ and $H_{12}=\delta_{1} / \delta_{2}=2.64 \pm 0.01$. Note that these values are based on four wall-normal profiles taken in a streamwise extent of $1750 \mathrm{~mm}$. The shape factor, being greater than the Blasius value of 2.59 , suggests that the present boundary layer is subjected to a weak adverse pressure gradient. Adding a Falkner-Skan deceleration parameter of $m=-0.015$, a theoretical shape factor of $H_{12}=2.64$ is obtained, which agrees with the experimental value of $2.64 \pm 0.01$. The corresponding theoretical $\delta_{1} / \delta$ and $\delta_{2} / \delta$ become 1.81 and 0.69 , respectively. These values can be obtained experimentally by introducing a virtual origin $\left(x_{0}\right)$ in order to compensate for the leading edge effects, which 



FIGURE 3. (Colour online) Normalized velocity contours for the configurations $\mathrm{C} 01, \mathrm{C} 03$ and C04 (column-wise, left to right) at different streamwise locations $\left(x-x_{M V G}\right) / h=6,47$, 203 and 445 (row-wise, top to bottom). The black and white contour lines show regions of velocity excess $(0.01,0.04,0.08,0.11,0.15)$ and deficit $(-0.08,-0.05,-0.03,-0.01,0)$, respectively. The grey lines correspond to the velocity contour lines $(0.1: 0.1: 0.9)$.

result in $x_{0}=-(21.5,43.1,86.2,172.3) \mathrm{mm}$, corresponding to the different streamwise locations given above.

\section{Characteristics of the streaky base flows and their scaling}

In order to understand the effect of the different parameters on the streamwise evolution of the boundary layer streaks, a parameter variation study is necessary. Moreover, it is of fundamental importance to find an appropriate scaling of the generated streaks. The results are important for future design purposes aimed at transition delay for skin-friction drag reduction.

\subsection{Boundary layer parameters and skin-friction coefficients}

As soon as the initially developed two-dimensional boundary layer flow impinges on the MVG array, the base flow starts to be modulated in a complex way and the two-dimensionality is going to be significantly altered. In figure 3 , the streaky base flow is shown for three different configurations (C01, C03 and C04), column-wise (left to right), and for four different downstream locations corresponding to $\left(x-x_{M V G}\right) / h=6,47,203$ and 445, row-wise (top to bottom). The figure shows $y z$ 
planes of normalized streamwise velocity contours as the grey lines $U(x, y, z) / U_{\infty}(x)$, while regions of velocity excess and deficit are depicted using black and white contour lines $\left(U(x, y, z)-U^{z}(x, y)\right) / U_{\infty}(x)$, respectively. The wall-normal coordinate is normalized by the local spanwise averaged displacement thickness, $\delta_{1}^{z}(x)$, while the spanwise coordinate is left unscaled in order to illustrate the effect of varying $\Lambda$ and $d$. All the measurements were performed over one complete wavelength $(\Lambda)$.

As shown in figure 3, high- and low-velocity streaks are formed in the boundary layer. The vortices being generated by each blade in a MVG pair, of counter-rotating configuration, are responsible for pushing high-speed fluid towards the wall in between the two blades and lifting low-speed fluid up from the wall on the sides. This gives rise to the high-speed region right behind the MVG pair and the low-speed regions at the sides. In the high-speed region, the boundary layer thickness becomes thinner than the two-dimensional boundary layer without the MVGs. This is clearly illustrated by the grey contour lines of constant velocity for the $\mathrm{C} 01$ configuration, in the left-hand column. From the figure, one may also conclude that when the distance between two blades of an MVG pair $(d)$ is relatively large, two individual high-low streaks are formed behind each blade (cf. C03, middle column). In this case, both the low- and high-speed streaks develop downstream and grow both in height (with the boundary layer), and in the spanwise direction, until they merge far downstream. Conversely, when the distance is relatively small, the two vortices from the MVG pair interact positively from the beginning and are able to produce higher streak amplitudes.

The symmetry is lost in the C04 configuration (right-hand column), where a row of single blades inclined in the same direction is used. In this case, the streaks appear inclined, probably due to the absence of a neighbouring vortex of opposite sign.

Another way to analyse the streaks in more detail is to look at the deviations of individual wall-normal velocity profiles from the spanwise averaged base flow, which locally shows regions of velocity excess and deficit. In figure 4, wall-normal profiles of $\left(U(x, y, z)-U^{z}(x, y)\right) / U_{\infty}$ are plotted for three different configurations, namely C05 (dotted line), C01 (dashed line) and C06 (solid lines), at four different normalized spanwise locations $\zeta=z / \Lambda$. The three configurations are similar except for a stepwise increase of $h$. As expected, a stronger modulation with increased $h$ (i.e. $\mathrm{C} 05 \rightarrow \mathrm{C01} \rightarrow \mathrm{C} 06)$ may be observed. At each streamwise location, one can move in $\zeta$ and observe the change from a velocity excess to a velocity deficit, confirming the presence of high- and low-velocity streaks. Moreover, the figure shows that the modulation becomes stronger initially and then decays in the streamwise direction; this may be observed for both the velocity excess as well as the velocity deficit profiles. The reason why the solid line (case C06) shows an almost flat profile at $\left(x-x_{M V G}\right) / h=445$ is that the flow has become turbulent. This happens when the MVG height Reynolds number, $R e_{h}=h U_{h} / v$ (where $U_{h}=U(y=h)$ ), exceeds a critical value, as is the case here. This will be discussed further in $\S 4.2$.

Having $y z$ plane measurements over one complete wavelength $(\Lambda)$ and at nine streamwise locations, it is possible to display the local integral boundary layer parameters, such as $\delta_{1}, \delta_{2}$ and $H_{12}$, in the $x z$ plane. Figure 5 shows the normalized values of these parameters for the $\mathrm{C} 01$ configuration. The boundary layer scale, $\delta$, is used for normalization, and the streamwise and spanwise directions are represented by the boundary layer scale Reynolds number, $\operatorname{Re}_{\delta}=\delta U_{\infty} / \nu$, and $\zeta$, respectively.

The figure shows that the contour plots are fairly symmetric. Small deviations could be due to misalignments or imperfections of the MVGs in the manufacturing process. Nevertheless, the contour plots show clearly that the maximum variation of $\delta_{1}$ in the spanwise direction appears around $R e_{\delta}=400-425$ (see figure $5 a$ ) and around 

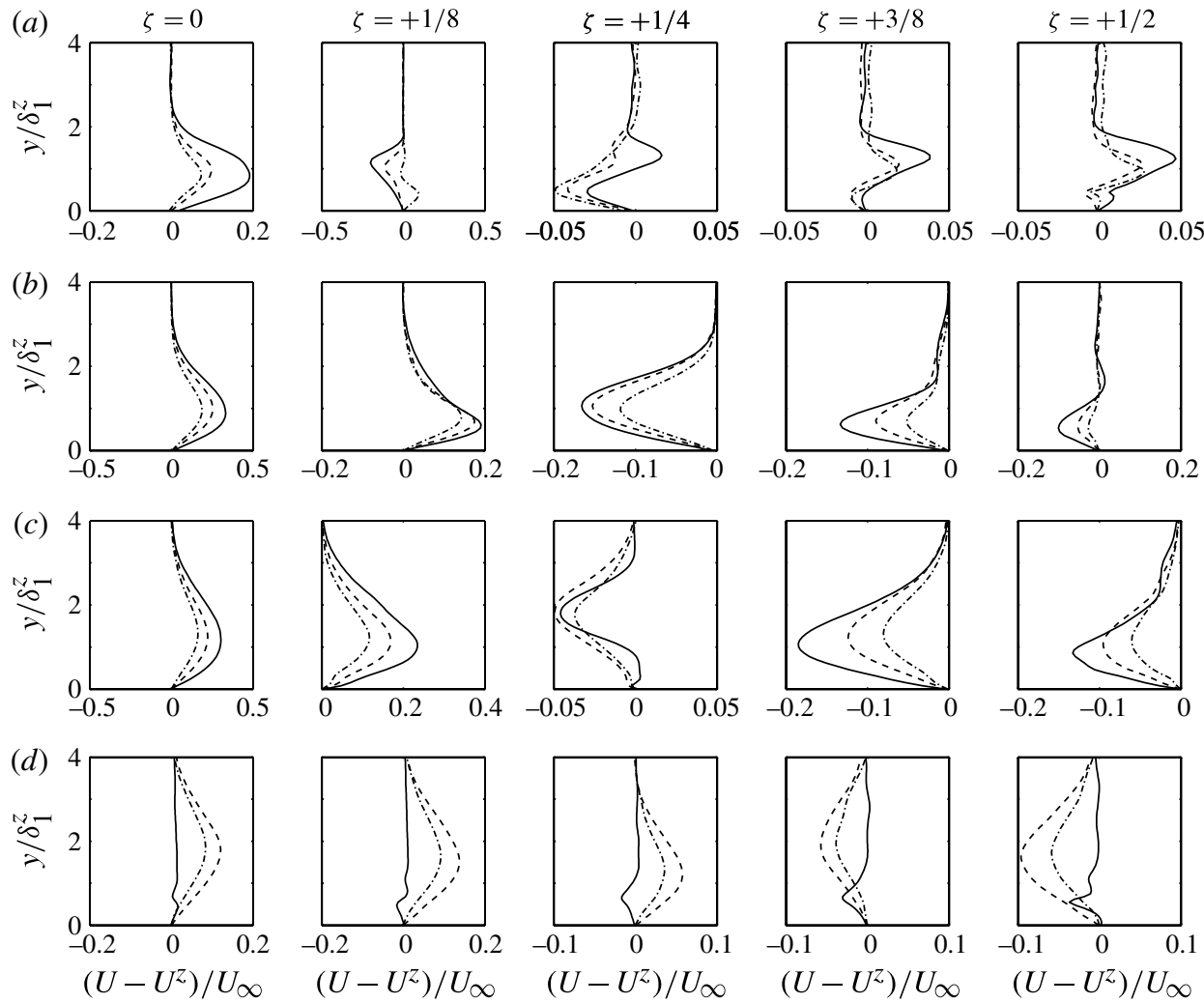

FIGURE 4. Local mean velocity deviation profiles from the spanwise averaged profile. The different columns correspond to different spanwise locations, $\zeta=(0,1 / 8,1 / 4,3 / 8,1 / 2)$, and the different rows to the downstream locations $\left(x-x_{M V G}\right) / h=6,47,203$ and 445 (top to bottom). The dotted, dashed and solid lines correspond to the configurations $\mathrm{C} 05, \mathrm{C} 01$ and C06, respectively.

$R e_{\delta}=500-525$ for $\delta_{2}$ (see figure $5 b$ ). Due to the high-speed region on the centre line behind an MVG pair (i.e. $\zeta=0$ ) the boundary layer is thinner and hence a region of lower values of $\delta_{1}$ and $\delta_{2}$ is observed. The shape factor in figure 5(c) reveals locally the stability of the streaky boundary layer, with a low value representing a fuller velocity profile compared to the reference case with $H_{12}=2.64$, and hence is more stable from a local stability point of view. Profiles with higher values of the shape factor are associated with inflection points and are unstable from an inviscid instability point of view. These are regions in which modal disturbance growth is expected to be strong.

In figure $5(d)$, the spanwise averaged parameters from figure $5(a-c)$ are plotted, where the dotted lines correspond to the Blasius boundary layer values. As can be noted, the spanwise averaged parameters from configuration C01 deviate only slightly from the Blasius parameters, and are representative for most of the other configurations, as illustrated in figure 6 , where the parameters from all of the configurations are plotted. The configurations that are distinguishable from the rest are, in particular, C06, C12 and C13. The deviations observed in these configurations are the result of streaks with excessively high amplitudes, which make the streaks 

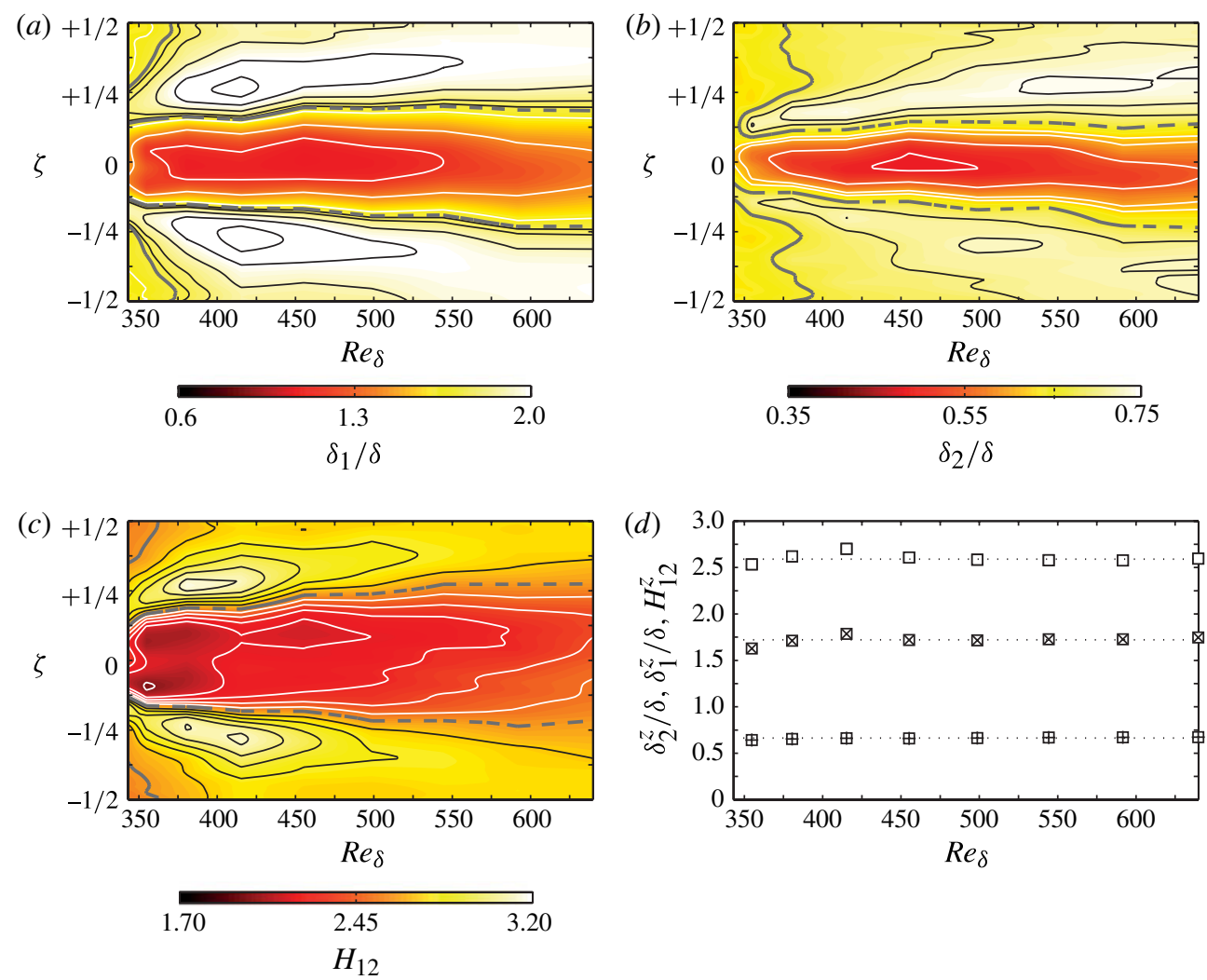

Figure 5. (Colour online) Contour plots of $(a) \delta_{1} / \delta,(b) \delta_{2} / \delta$ and $(c) H_{12}$, in the $x z$ plane, for configuration C01. (d) Spanwise averaged values $\left(\delta_{1}^{z} / \delta, \delta_{2}^{z} / \delta, H_{12}^{z}\right): \otimes$, from $(a)$; $\boxplus$, from $(b)$; $\square$, from $(c)$. The dotted lines correspond to the values of the Blasius boundary layer.

break down to turbulence. This is clearly illustrated by the significant drop in the shape factor.

An important aspect when striving after drag reduction by means of passive devices is to quantify the amount of induced drag that the devices cause. Here, in order to assess the local skin-friction coefficient, we use the momentum-integral equation for two-dimensional incompressible boundary layers:

$$
c_{f}(x)=2 \frac{\tau_{w}}{\rho U_{\infty}^{2}}=2\left[\frac{\mathrm{d} \delta_{2}^{z}}{\mathrm{~d} x}+\frac{1}{U_{\infty}} \frac{\mathrm{d} U_{\infty}}{\mathrm{d} x}\left(\delta_{1}^{z}+2 \delta_{2}^{z}\right)\right],
$$

where $\delta_{k}^{z}(x)$, with $k=1$ and 2 , are the spanwise average of the displacement thickness and the momentum thickness, respectively, defined as follows:

$$
\delta_{k}^{z}(x)=\int_{-1 / 2}^{1 / 2} \delta_{k}(x, z) \mathrm{d} \zeta
$$

Above, $\tau_{w}$ and $\rho$ correspond to the wall shear stress and the fluid density, respectively. In order to reduce the scatter of the data, which would appear in calculating $\mathrm{d} \delta_{2}^{z} / \mathrm{d} x$ from discrete points, we have here applied the same procedure as in Fransson \& Talamelli (2012) and made use of a curve-fitting technique and then calculated 


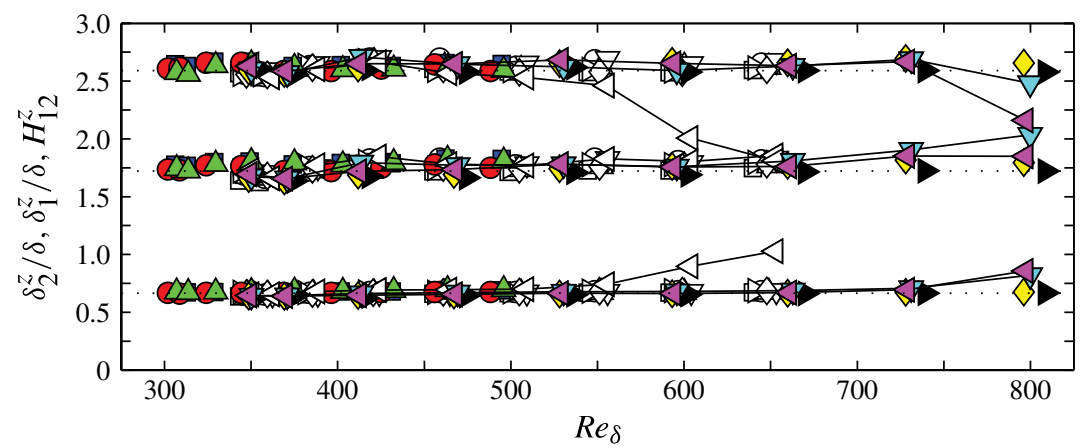

FIGURE 6. (Colour online) The spanwise averaged values of the boundary layer parameters, $\delta_{2}^{z} / \delta, \delta_{1}^{z} / \delta$ and $H_{12}^{z}$ for all configurations. The dotted lines indicate the corresponding Blasius parameter values.

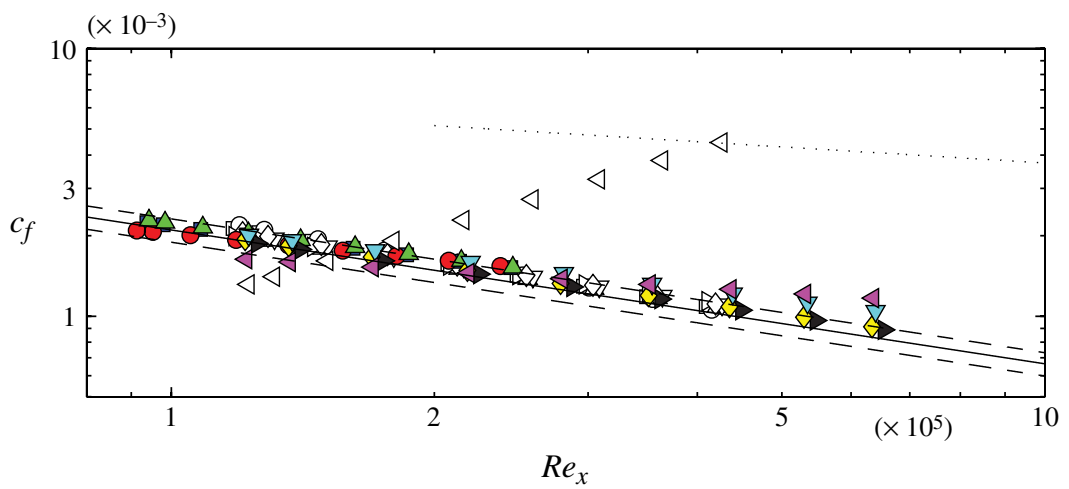

FIGURE 7. (Colour online) The skin-friction coefficient for all configurations. The solid line corresponds to the theoretical coefficient of the Blasius boundary layer and the dashed lines to $\pm 10 \%$ of this theoretical value. The dotted line corresponds to the empirical relation, $c_{f}=0.0592 \cdot R e_{x}^{-1 / 5}$, for a turbulent boundary layer.

the derivate in $x$ from a simple analytical function. In figure 7, the skin-friction coefficient is plotted versus the Reynolds number on the basis of the downstream distance. The figure shows that for most configurations, the skin-friction coefficient lies within $\pm 10 \%$ of the Blasius value, shown as a solid line. Configuration C06, which transitions to turbulence, clearly deviates from the Blasius value and approaches the empirical dotted line corresponding to a turbulent boundary layer. The figure shows that as long as the streaky boundary layers do not transition to turbulence, the skin-friction drag along the plate is comparable to the corresponding boundary layer without the MVGs mounted on the plate. This is confirmed by recent direct numerical simulations, performed on similar geometries by Camarri, Fransson \& Talamelli (2013), which show that the MVGs lead to an increase of the drag coefficient of only $2.5 \%$ over the considered plate length, with the force exerted directly on the MVG (not estimated in the experiments), which is two orders of magnitude lower and thus negligible. 


\subsection{The effect of $M V G$ parameters on the streak amplitude}

The conventional streak amplitude definition, which has been used in the past (including in Fransson \& Talamelli 2012), is based on the maximum velocity difference on a horizontal line above the wall and is hence based on two single-point measurements and often defined as a percentage of the free-stream velocity as follows:

$$
A_{S T}^{\max }(x)=\frac{1}{2 U_{\infty}} \max _{y}\{\Delta U(y)\}
$$

where

$$
\Delta U(y)=\max _{z}\{U(y, z)\}-\min _{z}\{U(y, z)\} .
$$

This streak amplitude definition is, however, inappropriate when one tries to relate it to the stabilization of disturbances, since it does not consider the mean streamwise velocity gradient in the spanwise direction, which is an essential ingredient of the observed stabilization (Cossu \& Brandt 2004). A second possibility is to use an integral-based streak amplitude definition, similar to the one used by White (2002) and White et al. (2005), which takes the spanwise periodicity of the streaks into account and is also defined as a percentage of the free-stream velocity:

$$
A_{S T}^{i n t}(x)=\frac{1}{U_{\infty}} \int_{-1 / 2}^{+1 / 2} \int_{0}^{\eta^{*}}\left|U(x, y, z)-U^{z}(x, y)\right| \mathrm{d} \eta \mathrm{d} \zeta,
$$

where

$$
\eta=\frac{y}{\delta}=\frac{y}{\sqrt{x v / U_{\infty}}} \text { and } \zeta=\frac{z}{\Lambda} .
$$

Differently from White (2002), which is based on the root-mean-square value of the velocity difference, this definition requires the 'absolute' modulation of the velocity field throughout the full cross-sectional plane, at each streamwise location, over one spanwise wavelength $(-0.5 \leqslant \zeta \leqslant+0.5)$ and data from the wall, up to some truncated wall-normal distance $\left(0 \leqslant \eta \leqslant \eta^{*}\right)$. In these experiments, this value is consistently taken to be $\eta^{*}=9$, which is well outside the boundary layer (recall that the Blasius boundary layer edge is at $\eta \approx 5$ ).

An illustrative example, emphasizing the need for an integral-based amplitude definition, is shown in figure 8, where the spanwise wavelength $\Lambda$ has been varied while the other parameters have been kept constant. The two streak amplitude definitions evolve downstream in similar ways behind the MVG array. The strong vortices, being generated at the location of the array, feed energy into the streamwise velocity component until they die out. Somewhere around that location, the streak amplitude peaks at a value denoted here as $A_{S T}^{p}$, and from there on it will start to decay because of viscous dissipation. However, the conventional amplitude in figure $8(a)$ is unable to differentiate the three cases from each other, since the MVG pairs are identical and the smallest $\Lambda$ is large enough to allow co-action of common up-flow among blades from neighbouring MVG pairs. Conversely, the integral-based amplitude definition in figure $8(b)$ makes a clear distinction, since it is sensitive to the extent of the modulated boundary layer over one spanwise wavelength, and shows a higher streak amplitude for decreasing $\Lambda$. Note that the same conventional amplitude, but distributed on a shorter spanwise wavelength, gives rise to larger spanwise velocity gradients $(\partial U / \partial z)$ and hence is expected to be more effective in damping the growth of disturbances. 

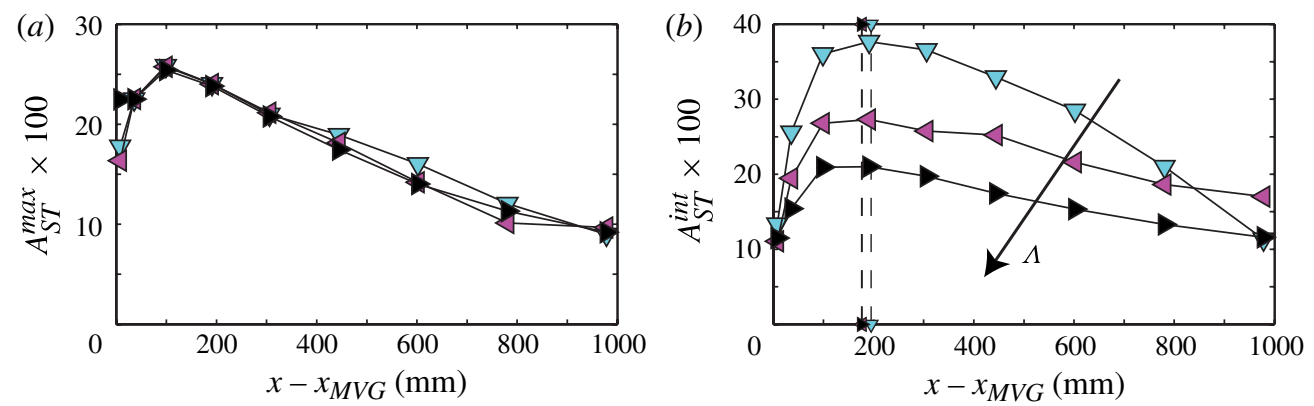

FIGURE 8. (Colour online) The evolution of the streak amplitude as a function of $\Lambda$ : $(a)$ with the conventional definition and $(b)$ with the integral based definition. $\nabla$, C12 with $\Lambda=13 \mathrm{~mm}$; 4 , C13 with $\Lambda=19.5 \mathrm{~mm} ; \rightarrow$, C14 with $\Lambda=26 \mathrm{~mm}$. The dashed lines highlight the locations of the streak amplitude peaks.

In figure 9, we present the integral evolution of the streak amplitude as a function of some different MVG configuration parameters as listed in table 1. Figure $9(a)$ shows the effect on the evolution of the streak amplitude of varying $U_{\infty}$ alone. It is clear that the peak streak amplitude increases with increasing $U_{\infty}$ and that the peak location in the streamwise direction, indicated by a dashed line, moves downstream. This result is in agreement with cases in which circular roughness elements were used as boundary layer modulators.

An interesting result is the effect of $h$ on the evolution of the streak amplitude. Similar to increasing $U_{\infty}$, an increase in $h$ contributes to the generation of stronger vortices and hence makes the streak amplitude peak further downstream (see figure $9(b)$ ). The evolution of streamwise streaks generated by circular roughness elements in a similar flow shows, on the other hand, no effect on the peak location in the streamwise direction: only an increase of the amplitude peak value was reported (White et al. 2005). The same effect has been shown in a spatially invariant boundary layer, i.e. in the asymptotic suction boundary layer (cf. Kurian \& Fransson 2011). There are several possible reasons for this discrepancy. First, the incoming spanwise vorticity, which is wrapped around the circular roughness elements (Fransson et al. 2004) and causes streamwise-orientated vortices, must initially overcome the velocity deficit region due to the wake behind the solid body. An increase in the roughness height sets up stronger vortices, but at the same time it creates a larger wake, which might balance the evolution of the streaky boundary layer. A second reason can be due to the change of shape in the frontal area of the MVGs when only $h$ is changed. Conversely, cylinders always present the same shape. In comparing cases $\mathrm{C} 05, \mathrm{C} 01$ and $\mathrm{C} 06$, for successively increasing $h$, but at a higher $U_{\infty}$ compared to figure $9(b)$, the same trends are observed, with the exception of C06. Indeed, for C06, the threshold streak amplitude, beyond which secondary instabilities set in and cause the onset of transition, has been exceeded. A $R e_{h}$ of 601 is obtained with an integral streak amplitude of $48 \%$ of $U_{\infty}$. This value of $R e_{h}$ can be compared to the critical $R e_{h}$ of around 547 for transition onset in the absence of any artificially forced TS waves (see Fransson \& Talamelli 2012).

Figure $9(c)$ shows the effect of varying the distance between the two blades in an MVG pair, $d$. Again, a larger distance between the blades (twice as long) is able to modulate the boundary layer over a larger region relative to the spanwise wavelength. This fact is highlighted in the integral-based measure over the maximum difference 

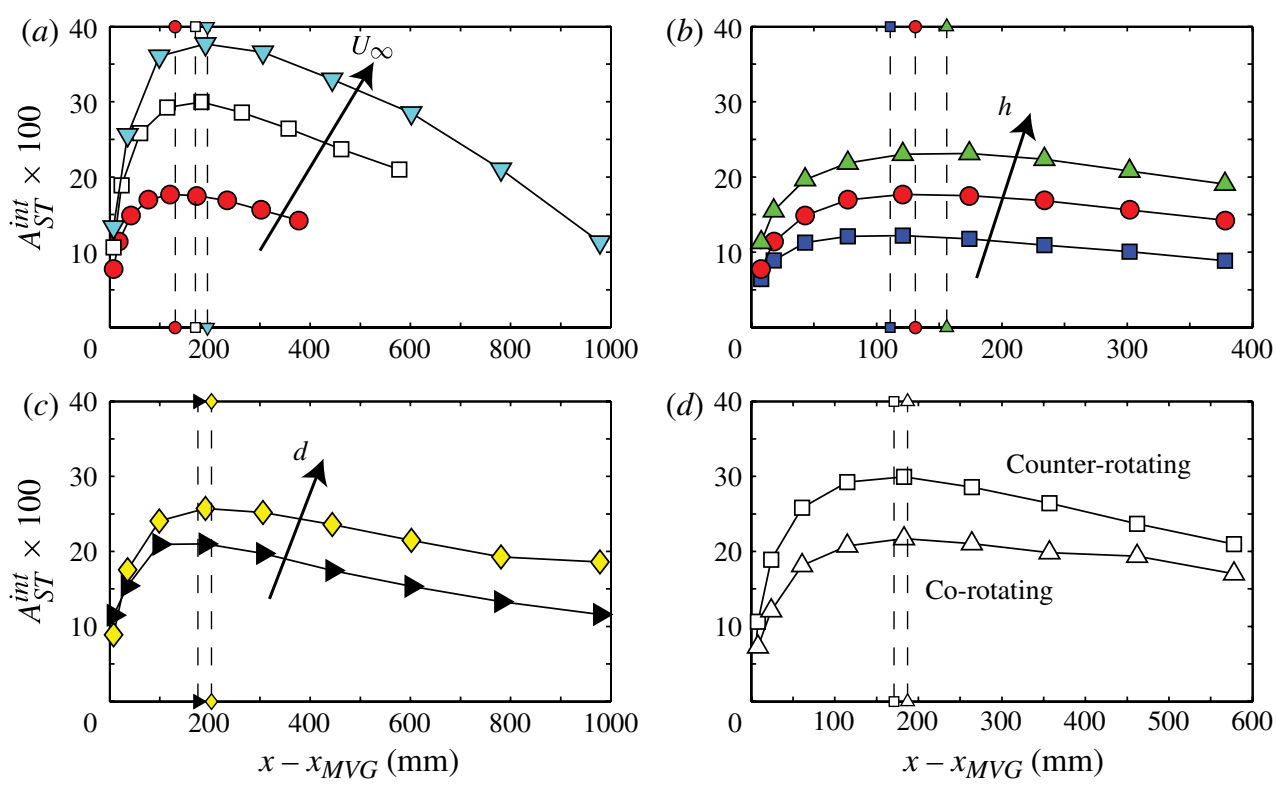

FIgURE 9. (Colour online) The evolution of the streak amplitude as a function of different parameters. (a) $U_{\infty}$ : •, C10, $U_{\infty}=6.0 \mathrm{~m} \mathrm{~s}^{-1} ; \square, \mathrm{C} 01, U_{\infty}=7.7 \mathrm{~m} \mathrm{~s}^{-1} ; \nabla, \mathrm{C} 12$, $U_{\infty}=8.2 \mathrm{~m} \mathrm{~s}^{-1}$. (b) $h: \square, \mathrm{C} 09, h=1.1 \mathrm{~mm} ; \boldsymbol{O}, \mathrm{C} 10, h=1.3 \mathrm{~mm} ; \boldsymbol{\nabla}, \mathrm{C} 11, h=1.5 \mathrm{~mm}$. (c) $d$ : $\triangleright, \mathrm{C} 14, d=3.25 \mathrm{~mm} ; \diamond, \mathrm{C} 15, d=16.25 \mathrm{~mm}$. (d) counter versus co-rotating vortices: $\square$, C01, counter-rotating setting; $\triangle, \mathrm{C} 04$, co-rotating setting. The dashed lines highlight the locations of maximum amplitudes.

between velocity excess and deficit, which may take place very locally but may leave a large region of the spanwise wavelength untouched.

In figure $9(d)$, we show the evolution of the streak amplitude of the only configuration with a co-rotating setting ( $\mathrm{C} 04)$ compared to an $\mathrm{MVG}$ pair configuration with a counter-rotating setting (as in all other cases). The counter-rotating setting is the $\mathrm{C} 01$ case and the only difference in $\mathrm{C} 04$ is that every second blade in the C01 configuration has been removed, rendering half as many MVG blades in the C04 case compared to the C01 case. Despite this, the evolution of the streak amplitude does not differ significantly apart from the streak amplitude peak values, which change from $\sim 30$ to $22 \%$ for $\mathrm{C} 01$ and $\mathrm{C} 04$, respectively. The base flow will be asymmetric in the $y z$ plane for the $\mathrm{C} 04$ case, due to the generation of a single vortex by a blade inclined at $15^{\circ}$ against the streamwise direction (see the right-hand column of figure 3 ). It is of note that the single vortex is likely to induce a secondary vortex of opposite circulation, as measured and reported by Lögdberg, Fransson \& Alfredsson (2009). This induced vortex is weaker than the primary one and is unable to straighten up the asymmetry of the base flow. This asymmetry should, however, not be a problem for the stabilization of disturbances, even though it is expected to be somewhat less effective due to the weaker spanwise velocity gradient on one of the sides over a spanwise wavelength.

\subsection{Scaling of streamwise streaks}

The streak amplitude curves for all configurations (C01-C17), using the integral-based definition, are summarized in figure 10. The horizontal and vertical grey-filled regions 




FIGURE 10. (Colour online) The evolution of the streak amplitude in the streamwise direction for all cases C01-C17. See table 1 for symbols. The dark grey area indicates the variation both in the maximum streak amplitude and in the location in the streamwise direction at which these maxima appear.

represent the variation of $A_{S T}^{i n t}$ from the minimum to the maximum of $A_{S T}^{i n t, p}$ for all cases and their corresponding streamwise locations, respectively. The $A_{S T}^{\text {int } p}$ values range from 12 to $61 \%$ and the streamwise locations at which the streak amplitude maxima appear are in the range $\left(x-x_{M V G}\right)=77$ to $264 \mathrm{~mm}$. In this subsection we seek, without prejudice, an empirical streak amplitude scaling. From inspection of the streak amplitude shape (cf. figure 10), consisting of a seemingly initial algebraic growth from $x=x_{M V G}$ followed by an exponential decay of the streak amplitude, our basis will be the simple function

$$
\mathscr{F}=\left\{A_{S T}^{i n t} / A_{S T}^{i n t *}\right\}=\xi \mathrm{e}^{-\xi},
$$

where $A_{S T}^{i n t *}$ is the normalization streak amplitude. As pointed out by Luchini (1996), a small-amplitude three-dimensional instability initially grows and then decays, both algebraically and independent of the Reynolds number, in a developing boundary layer. On the other hand, the MVGs generate strong vortices with a circulation proportional to the lift force on the elements, which are able to modulate the boundary layer into high-amplitude streaks. The circulation decays exponentially as shown in Lögdberg et al. (2009), and here the base flow is assumed to recover accordingly, motivating our ansatz of exponential decay of the high-amplitude streaks. It is worth mentioning that the ansatz of algebraic decay has been tested but does not lead to equally good final results. Furthermore, previously suggested models based on the energy (see e.g. White et al. 2005, and references therein) were also tested, but with less satisfactory fits compared to ansatz (4.6).

We now seek the variable $\xi$ in (4.6) in the general form

$$
\xi=\left[\mathscr{C}_{\xi}^{i}\left(x / x_{M V G}-1\right)\right]^{\mathscr{C}}{ }_{\xi}^{i i},
$$

and $A_{S T}^{\text {int* }}$ such that it peaks at a value of $\exp \{-1\} \approx 0.37$ at $\xi=1$. In minimizing the error between the data and the function (4.6) by means of a least-squares fit method, one may determine the coefficients $\mathscr{C}_{\xi}^{i}$ and $\mathscr{C}_{\xi}^{i i}$ in $\xi$ (cf. equation (4.7)) and the normalization amplitude $A_{S T}^{i n t *}$ in (4.6) for each case. The result is plotted in 




FIgURE 11. (Colour online) The shape of the evolution of the streak amplitude. The solid line corresponds to (4.6). All cases are individually fitted to (4.6), giving unique values of $A_{S T}^{i n t *}, \mathscr{C}_{\xi}^{i}$ and $\mathscr{C}_{\xi}^{i i}$ in (4.6) and (4.7).

figure 11 and shows that the evolution of the streak amplitude behind MVGs has a general shape, which can be described by the function (4.6). This is not a trivial result, since the data cannot be forced to take any shape. Note that for C06 and C12, which both transition to turbulence due to the TS wave forcing in connection with relatively high-amplitude streaks, only data points that do not appear to be affected by the transition were used in the least-squares fitting procedure. This is why a few downstream data points from each of the two cases C06 and C12 lie significantly below the curve described by function (4.6) in figure 11.

Next, we empirically seek parameter combinations, based on both geometrical parameters and boundary layer parameters, which best correlate with the coefficients $\mathscr{C}_{\xi}^{i}, \mathscr{C}_{\xi}^{i i}$ and $A_{S T}^{i n t *}$. The results are shown in figure $12(a-c)$, respectively. For $\mathscr{C}_{\xi}^{i}$, we have identified the ratios $(h / \delta)$ and $(\Lambda / d)$ to be leading quotients, with powers of $\alpha$ and $\beta$, respectively. These exponents can again be determined using a least-squares fit technique applied to all the cases, which results in $\alpha=-7 / 5$ and $\beta=2 / 5$. For $\mathscr{C}_{\xi}^{i i}$, an averaged value of 0.55 comes out when all cases are considered. For the sake of illustration in figure $12(b)$, this coefficient is plotted versus the MVG height Reynolds number, $R e_{h}$. Here, we choose $\mathscr{C}_{\xi}^{i i}=1 / 2$ with the aim of obtaining a neat and simple expression for the variable $\xi$, which results in

$$
\xi=\sqrt{\left(\frac{h}{\delta_{1}}\right)^{\alpha}\left(\frac{\Lambda}{d}\right)^{\beta}\left(x / x_{M V G}-1\right)} .
$$

The above expression for $\xi$ suggests that the streak energy initially grows in linear proportion to the downstream distance, since a McLaurin expansion of (4.6) gives $\mathscr{F} \approx \xi$. In the limit $\xi \rightarrow \infty$, the viscous dissipation, in terms of exponential decay, prevails over the algebraic growth and nullifies the streak amplitude, with a full recovery to the originally two-dimensional boundary layer.

The normalization amplitude $A_{S T}^{i n t *}\left(=\exp \{-1\} \times A_{S T}^{i n t, p}\right)$ needs special consideration. Looking at the available cases, we learn that $\Lambda / d$ again plays a leading role, giving a maximum amplitude for $\Lambda / d \approx 4$. Furthermore, by looking at figure $9(a, b)$, it is clear that both $U_{\infty}$ and $h$ ought to take part in the amplitude scaling. On the basis of the present data, we here propose $R e^{2}=\left(h U_{\infty} / v\right)^{2}$. It should be pointed out that, logically, $R e_{h}=h U(y=h) / v$ with arbitrary power was tested, but the best scaling was found 

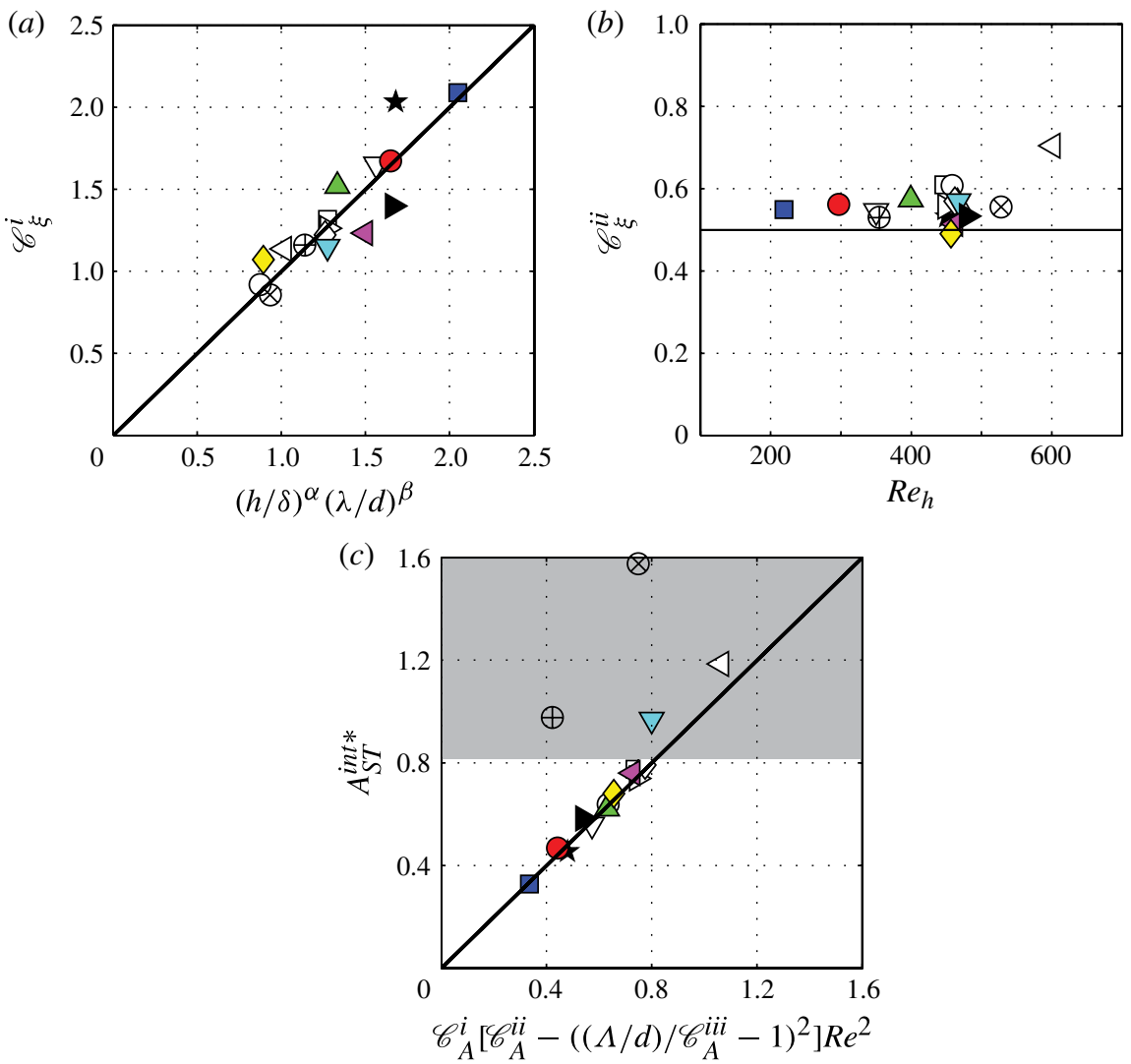

FIGURE 12. (Colour online) The coefficients $\mathscr{C}_{\xi}^{i}, \mathscr{C}_{\xi}^{i i}$ and $A_{S T}^{i n t *}$, appearing in (4.6) and (4.7), are plotted in $(a-c)$, respectively, versus the proposed scaling. The coefficients have been determined by means of a least-squares fit of the data to (4.6). The additional coefficients are summarized in table 2 . See the running text for the grey region in $(c)$.

with $R e^{2}$, and the final proposed scaling of the streak amplitude becomes

$$
A_{S T}^{i n t *}=\mathscr{C}_{A}^{i}\left[\mathscr{C}_{A}^{i i}-\left(\frac{\Lambda / d}{\mathscr{C}_{A}^{i i i}}-1\right)^{2}\right]\left(\frac{U_{\infty} h}{v}\right)^{2} .
$$

The coefficients $\mathscr{C}_{A}^{i}, \mathscr{C}_{A}^{i i}$ and $\mathscr{C}_{A}^{i i i}$ were determined in a least-squares fit sense to the data and the result is shown in figure $12(c)$. The grey region in this figure corresponds to peak streak amplitudes of $A_{S T}^{\text {int,p }}$ beyond $30 \%$. The data points in this region deviate from the relation (4.9), while the data below the grey region obey the relation (4.9) perfectly. This leads us to the conclusion that nonlinear effects in the evolution of the streamwise streaks become important above $30 \%$ using the integral-based streak amplitude definition, and hence deviate from the proposed scaling.

In figure 13 , we test the proposed scaling on all the data satisfying $A_{S T}^{\text {int, } p}<0.30$, using $\xi$ and $A_{S T}^{\text {int* }}$ given in (4.8) and (4.9), respectively. All chosen coefficients based on the least-squares fit method are summarized in table 2. The solid black line in figure 13 corresponds to the general streak amplitude evolution curve given by (4.6). We may conclude that the data we have available, having neglected C06, C12, C16 


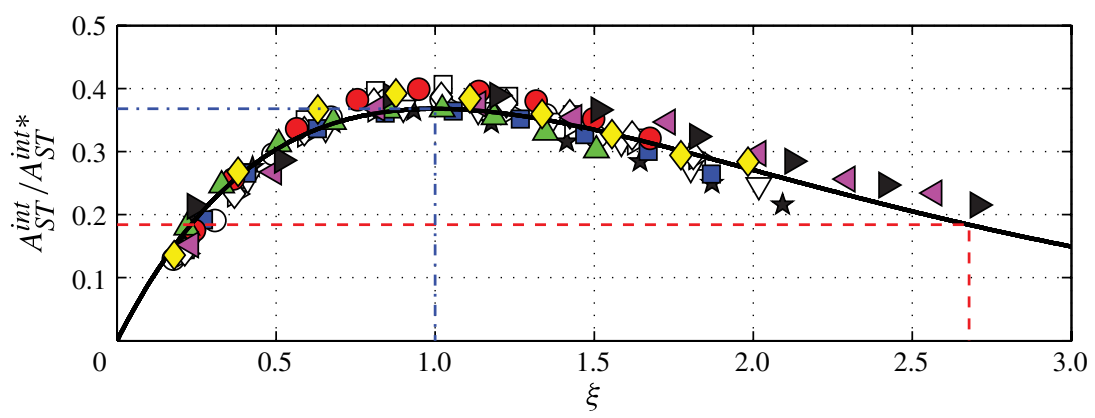

FIgURE 13. (Colour online) The evolution of the streak amplitude using the scaling proposed in (4.6), (4.8) and (4.9) for all configurations below $A_{S T}^{i n t, p}=0.30$. Above this threshold, amplitude value nonlinear effects seem to become important. The solid line corresponds to (4.6); for the dash-dotted and dashed lines, see the running text.

$$
\begin{array}{cccccc}
\alpha & \beta & \mathscr{C}_{\xi}^{i i} & \mathscr{C}_{A}^{i} & \mathscr{C}_{A}^{i i} & \mathscr{C}_{A}^{i i} \\
-7 / 5 & 2 / 5 & 1 / 2 & 7.25 \times 10^{-7} & 2.3 & 4.2
\end{array}
$$

TABLE 2. Coefficients appearing in the proposed scaling of streamwise streaks and determined by means of a least-squares fit to the data.

and $\mathrm{C} 17$ due to nonlinear effects, follow the proposed scaling satisfactorily. This figure should be compared with figure 10 without the four highest streak amplitude cases.

The dash-dotted line in figure 13 indicates the location of the peak amplitude corresponding to $A_{S T}^{i n t, p}=\exp \{-1\} \times A_{S T}^{i n t *}$ for $\xi=1$. An implicit expression for $\xi$ can readily be derived for a $50 \%$ drop in $A_{S T}^{\text {int, } p}$ (i.e. to $\exp \{-1\} \times A_{S T}^{i n t *} / 2$ ), which solves to $\xi=2.678$ (indicated by the dashed line) and gives an idea of how fast the streak amplitude decays. Substituting these $\xi$ values in the rewritten expression of (4.8) given below, one can predict the corresponding physical downstream locations on the basis of the actual MVG configuration:

$$
\frac{x}{x_{M V G}}=1+\left(\frac{\delta_{1}}{h}\right)^{\alpha}\left(\frac{d}{\Lambda}\right)^{\beta} \times \xi^{2},
$$

where $\alpha$ and $\beta$ are given in table 2 .

In figure 14, the normalized wall-normal location at which the peak value of the spanwise integral-based streak amplitude measure, $\max \left\{A_{S T}^{z}(y)\right\}$, appears is plotted versus the downstream location $\xi$ for all configurations. The data are scattered when plotted in their dimensional form (not shown), but become more collected when the $\xi$ variable and the spanwise averaged displacement thickness are used to normalize the data, as shown in figure 14. The two configurations that deviate significantly from the rest are $\mathrm{C} 03$ and $\mathrm{C} 15$, both with $\Lambda=26$ and $d=16.25$. This configuration differs from the others in the sense that the two vortices closest to each other have common up-flow as opposed to common down-flow. At the streak amplitude peak, i.e. at $\xi=1$, the data are collected around $y_{\max } / \delta_{1}^{z}=0.6$ above the wall, as indicated by the dashed lines in the figure. Downstream of this position, the data are somewhat 


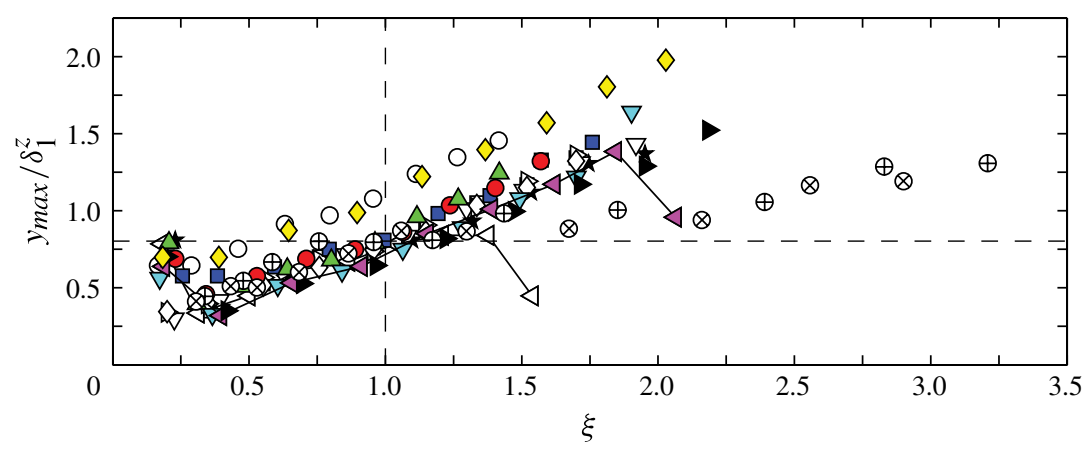

FIGURE 14. (Colour online) The normalized wall-normal location $\left(y_{\max } / \delta_{1}^{z}\right)$ where $\max \left\{A_{S T}^{z}(y)\right\}$ appears plotted versus the downstream location $\xi$ for all configurations.

spread out, emphasizing the importance of the geometrical parameters of the MVGs on the evolution of the vortex core.

\section{Passive laminar flow control using MVGs}

Miniature vortex generators have previously been shown to be effective in creating streaks with amplitudes up to $32 \%$ of $U_{\infty}$. Here, we grasp the nettle by setting up a fairly realistic flow configuration, i.e. by generating TS waves upstream of the MVG array, keeping the full and complicated receptivity process of the incoming TS wave by the MVG array. We believe that this configuration really challenges the previously reported control strategy.

\subsection{Tollmien-Schlichting waves generated upstream of the $M V G$ array}

In this section, we report results on the development of linear disturbances generated as described in $\$ 2.2$. In the linear regime, the growth rate of the disturbances is independent of the initial forcing amplitude, which was validated here by comparing the growth curves from the configurations $\mathrm{C} 01, \mathrm{C} 07$ and $\mathrm{C} 08$ (not shown). These three configurations have, indeed, the same setting apart from different initial forcing amplitudes. Note that the last column in table 1 shows the TS wave amplitude as a percentage of the free-stream velocity, for the reference case at branch II of the neutral stability curve.

Cross-sectional contour plots of the normalized TS wave amplitude distribution filtered around the forcing frequency, $A_{T S}(x, y, z) / U_{\infty}$, are shown in figure 15 for four downstream locations $\left(x-x_{M V G}\right) / h=6,47,203$ and 445 (row-wise, top to bottom) and for three different configurations C01, C03 and C06 (column-wise, left to right). The wave frequencies $F$, defined in $\S 2.2$, are within $6 \%$ between the three configurations (see table 1). It is clear that the different configurations affect the TS wave differently and that in $\mathrm{C} 06$, the configuration furthest to the right in figure 15, the laminar flow control concept fails, since transition to turbulence takes place between the second to last and last downstream location: this reveals the weak point of this passive flow control method (Shahinfar et al. 2012). When the gradients of the mean streamwise velocity component become large due to the high streak amplitudes, the base flow becomes susceptible to secondary inviscid instabilities of sinuous or varicose type, depending on whether an inflection point in the mean streamwise velocity component 



FIGURE 15. (Colour online) Cross-sectional contour plots of the local TS wave amplitude for the configurations $\mathrm{C} 01, \mathrm{C} 03$ and $\mathrm{C} 06$ (column-wise, left to right), at different streamwise locations $\left(x-x_{M V G}\right) / h=6,47,203$ and 445 (row-wise, top to bottom). All values are normalized with $U_{\infty}$ and $F$ is $\sim 135$.

appears first in the wall-normal or spanwise direction, respectively (Andersson et al. 2001).

In figure 16, local wall-normal TS wave amplitude profiles for the C01 configuration are shown. The streamwise locations are the same as the four downstream locations depicted in figure 15. Figure 16(a) shows the reference configuration without MVGs, and $(b),(c)$ and $(d)$ with MVGs at the spanwise locations corresponding to the low (b) and high $(d)$ speed streaks of the base flow and, in between them, in the shear layer $(c)$. An interesting observation is that the TS wave amplitude initially grows more in the low-speed streak compared to the reference case, where it is significantly damped. In the high-speed streak, the amplitude does not seem to be as high as in the low-speed streak, but it appears to decay somewhat more slowly than in the low-speed streak. The shear layer displays a TS wave amplitude distribution in the wall-normal direction shaped as an $\mathrm{M}$ at some downstream location, with an inner and an outer peak. The M-shaped TS wave profile has previously been reported for circular roughness elements, but solely in the low-speed streak. It is of note that the TS waves in the streaky base flow behind roughness elements behave rather differently, with the strongest TS wave growth in the high-speed streak, which is somewhat counterintuitive 


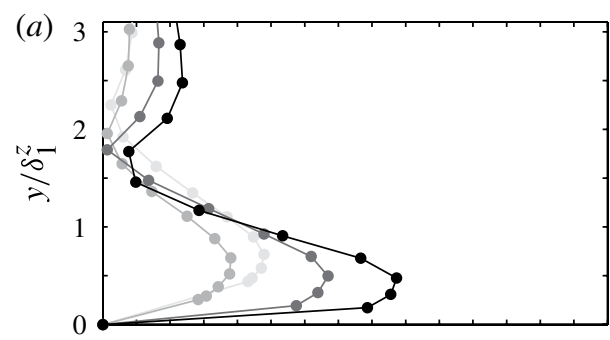

(b)
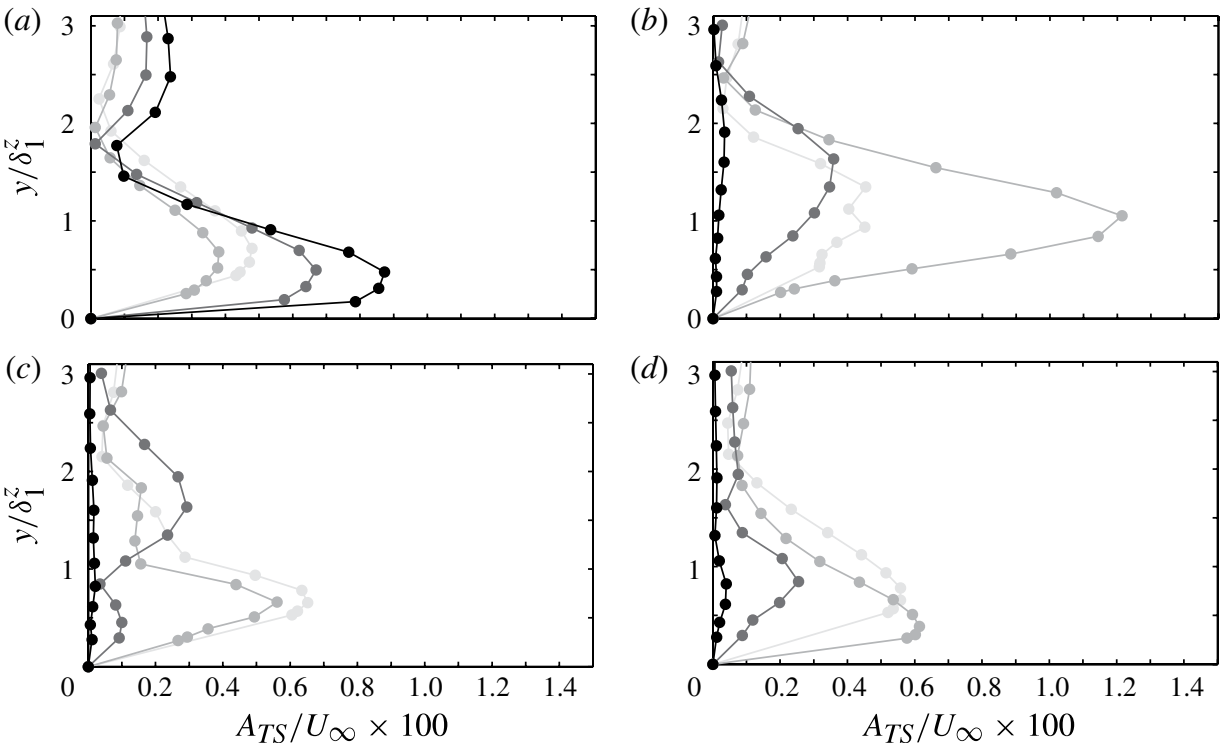

(d)

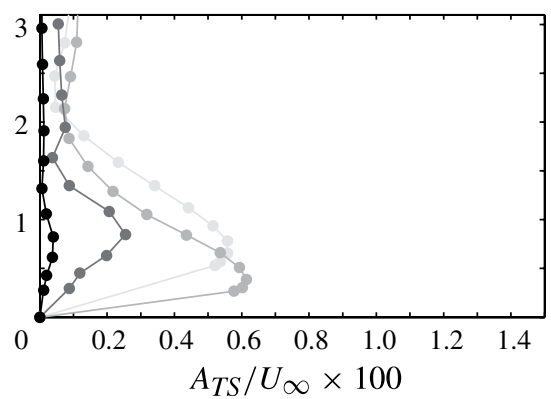

FIGURE 16. Local wall-normal TS wave amplitude profiles for configuration C01. The downstream streamwise distance is indicated by successively darker symbols, with the actual downstream locations being the same as depicted in figure 15: $(a)$ reference case; $(b)$ lowspeed streak; $(c)$ in between high and low-speed streaks; $(d)$ high-speed streak.

since the mean velocity profile is locally more stable there (Fransson et al. 2005). The explanation must be the presence of the near-wake region behind the roughness elements.

As pointed out in Fransson et al. (2005), where different TS wave amplitude measures were compared, it is necessary to integrate an entire cross-sectional plane over one spanwise wavelength of the streaky base flow (i.e. $\Lambda$ ) and up to some truncated wall-normal distance above the wall, $\eta^{*}$, in order to accomplish a fair comparison with the TS wave amplification in a two-dimensional reference base flow. Similarly as in Fransson et al. (2005), we here define the local streamwise TS wave amplitude as follows:

$$
A_{T S}^{y z}(x)=\int_{-1 / 2}^{+1 / 2} \int_{0}^{\eta^{*}} \frac{A_{T S}(x, y, z)}{U_{\infty}} \mathrm{d} \eta \mathrm{d} \zeta,
$$

where $\eta^{*}$ is the same as in (4.4). The actual choice of $\eta^{*}$, be it 7,8 or 9 , will have a negligible influence on the TS amplification curves.

With this integral measure, we next compare the relative amplification curves of the TS waves with different MVG configurations with the two-dimensional reference base flow. In figures 17 and 18, the TS amplitude, (5.1), is plotted and normalized with its most upstream value, $A_{T S, 0}^{y z}$, in contrast to the classical way of plotting TS growth curves, i.e. normalized with the amplitude at branch I of the neutral stability curve. In these figures, the corresponding reference growth curve is plotted using asterisks (*) and shows the decay of the amplitude until the wave enters the neutral stability curve at branch I. Here, it starts to amplify and continues doing so until it exits the stability curve at branch II. Figure $17(a, b)$ shows the effect of increased MVG height $(h=1.3,1.1$ and $1.5 \mathrm{~mm})$, implying an increase in streak amplitude with a 



FIgURE 17. (Colour online) The effect of $h$ on the TS amplitude amplification curve. (a) Configurations $\mathrm{C} 09, \mathrm{C} 10$ and $\mathrm{C} 11$, with $U_{\infty} \approx 6 \mathrm{~m} \mathrm{~s}^{-1}$ and $F \sim 175$. (b) Configurations C05, C01 and C06, with $U_{\infty} \approx 7.8 \mathrm{~m} \mathrm{~s}^{-1}$ and $F \sim 135$. $*$, Two-dimensional reference base flow. For symbols, see table 1 .


FIgURE 18. (Colour online) The effect of $d$ and $\Lambda$ on the TS amplitude amplification curve. (a) Configurations $\mathrm{C} 12, \mathrm{C} 13$ and $\mathrm{C} 14$, with varying $\Lambda$ and $F \sim 100$. (b) Configurations $\mathrm{C} 14$ and $\mathrm{C} 15$, with varying $d$ and $F$ around 100 . In both $(a)$ and $(b), U_{\infty} \approx 8 \mathrm{~m} \mathrm{~s}^{-1}$. $*$, Two-dimensional reference base flow. For symbols, see table 1 .

fixed spanwise wavelength, on the amplification curves for two different free-stream velocities, i.e. $\sim 6.0$ and $7.8 \mathrm{~m} \mathrm{~s}^{-1}$, respectively. A general remark is that the strong amplification of the reference case at branch II is successively damped, reducing the growth factor, which becomes negative for high enough streak amplitudes. On the other hand, an initial amplification is observed right behind the MVG array, with a peak in the TS amplification around the streak amplitude peak, which is close to the location of branch I of the reference TS growth curve. This initial growth is enhanced with increasing $h$, which is worrying for control purposes, since it might cause a 
bypass transition, which is the case for the highest streak amplitude configuration, C06, in figure $17(b)$.

The effects on the TS amplification curves of varying $\Lambda$ and $d$ are shown in figure $18(a, b)$, respectively. In $(a)$, with the configurations $\mathrm{C} 12, \mathrm{C} 13$ and $\mathrm{C} 14$, the free-stream velocity is about the same, $U_{\infty} \approx 8 \mathrm{~m} \mathrm{~s}^{-1}$, but with $A_{S T}^{\text {int,p }}=37.7,27.3$ and $21.0 \%$, respectively, for increasing $\Lambda$. The damping effect on the TS wave is clear in the figure and it is seen that the higher the streak amplitude is, the stronger is the stabilizing effect. However, the relatively high-amplitude streaky base flow configurations, namely $\mathrm{C} 12$ and C13, together with the presence of the TS waves, give rise to an onset of transition, which is manifested by a strong relative amplification on the two furthest downstream measured points.

An interesting observation is that the initial amplification with a peak around branch I of the reference case is not affected by a change in $\Lambda$. Both the initial growth and the peak value are about the same, which suggests that the initial amplification can be related to the conventional streak amplitude, which remains the same for different values of $\Lambda$.

In figure $18(b)$, the effect on the TS amplification curve is shown for the configurations $\mathrm{C} 14$ and $\mathrm{C} 15$, both with the same large value of $\Lambda$ but with a change in the distance between the blades, $d$. The larger $d$ value (C15) gives a peak streak amplitude of $A_{S T}^{\text {int,p }}=25.7 \%$, with a configuration that can be seen as individual blades with initially negligible interactions with neighbouring blades. The other configuration in figure $18(b), \mathrm{C} 14$, with a clear MVG pair (i.e. a small value of $d$ ) with co-acting vortices will generate a higher local streak amplitude but a lower amplitude considering the integral measure $\left(A_{S T}^{\text {int }, p}=21.0 \%\right)$ since the distance to the neighbouring MVG pair is relatively long. Here, it is clear that an increased value of the integral-based streak amplitude measure correlates with the attenuation of the TS wave due to the inherent consideration of the spanwise gradient of the streamwise mean velocity component.

In figure 19, a direct comparison is made between counter-rotating and co-rotating vortices. It must be pointed out that the latter configuration (C04) is obtained by removing one blade from each $\mathrm{MVG}$ pair, i.e. it contains half as many blades as the base configuration $\mathrm{C} 01$. Not only do the two configurations have close to the same free-stream velocity, but they also have about the same non-dimensional TS wave frequency. An interesting observation in figure 19 is that, despite the significantly lower peak streak amplitude in $\mathrm{C} 04\left(A_{S T}^{\text {int }, p}=21.7 \%\right)$ compared to C01 $\left(A_{S T}^{i n t, p}=29.9 \%\right)$, there is a minor difference in the TS wave amplification curves. Both streaky base flows act very convincingly in attenuating the TS wave. One should keep in mind that a lower peak streak amplitude significantly reduces the initial growth of the local skin-friction coefficient (cf. Fransson \& Talamelli 2012), implying that the co-rotating configuration can actually be seen as the more attractive one of the two.

In the following, we define a TS wave attenuation factor $(\kappa)$ as the ratio between the TS wave amplitude with and without the MVG array. That is, the TS amplitude in the streaky base flow case over the TS amplitude in the reference base flow at the location of branch II will define $\kappa$ as follows:

$$
\kappa=\frac{\left[A_{T S}^{y z}\right]_{3 D}^{I I}}{\left[A_{T S}^{y z}\right]_{2 D}^{I I}} .
$$

From figure 20, one can see that all cases except one display an attenuation of the TS wave, with $\kappa<1$, in the presence of the streaky base flow as compared with the two- 


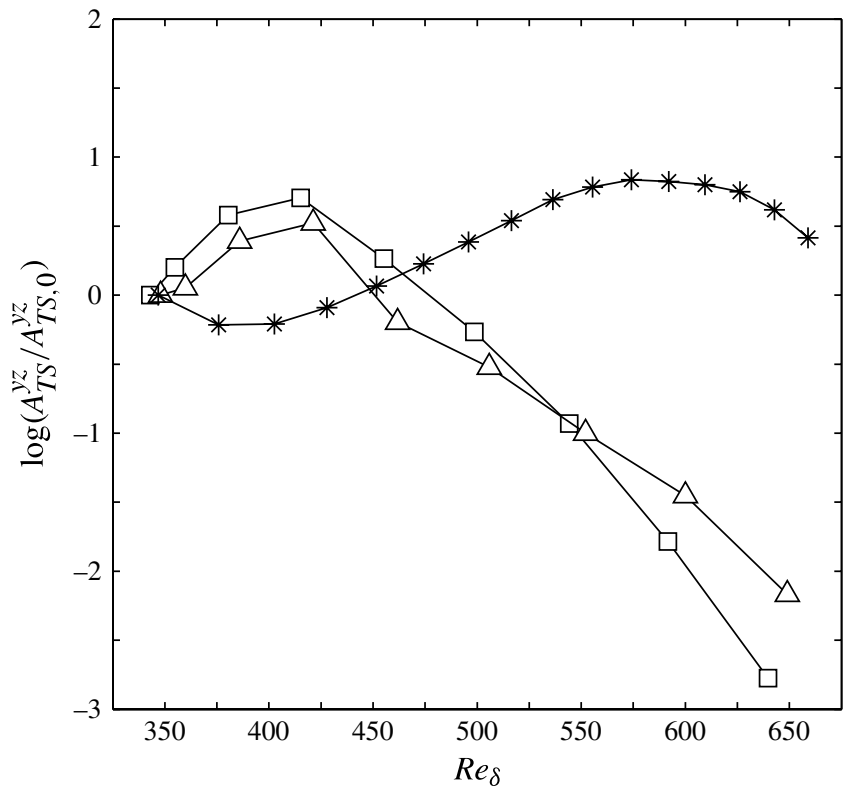

FIGURE 19. The effect and comparison of the presence of co-rotating vortices (C04) with counter-rotating vortices (C01) on the TS amplitude amplification curve. $*$, Two-dimensional reference base flow. For symbols, see table 1 .

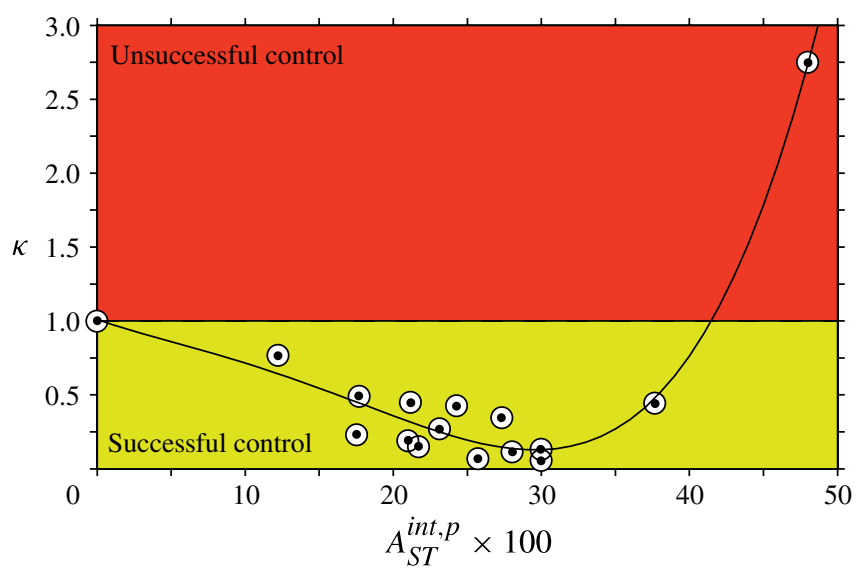

FIgURE 20. (Colour online) The TS wave attenuation factor $(\kappa),(5.2)$, versus the peak streak amplitude $\left(A_{S T}^{i n t, p}\right)$. The solid line is a fourth-order polynomial curve fit to the data in order to emphasize the trend.

dimensional reference base flow. We can conclude that the higher the streak amplitude, the stronger is the stabilization effect, at least up to some threshold amplitude around $30 \%$, above which the trend changes. Increasing the streak amplitude further may lead to bypass transition with unsuccessful control in terms of an advanced transition location as compared to the two-dimensional reference base flow. This is the case with configuration C06, with $A_{S T}^{\text {int }, p}$ of $48 \%$, showing the only unsuccessful case in figure 20 . 

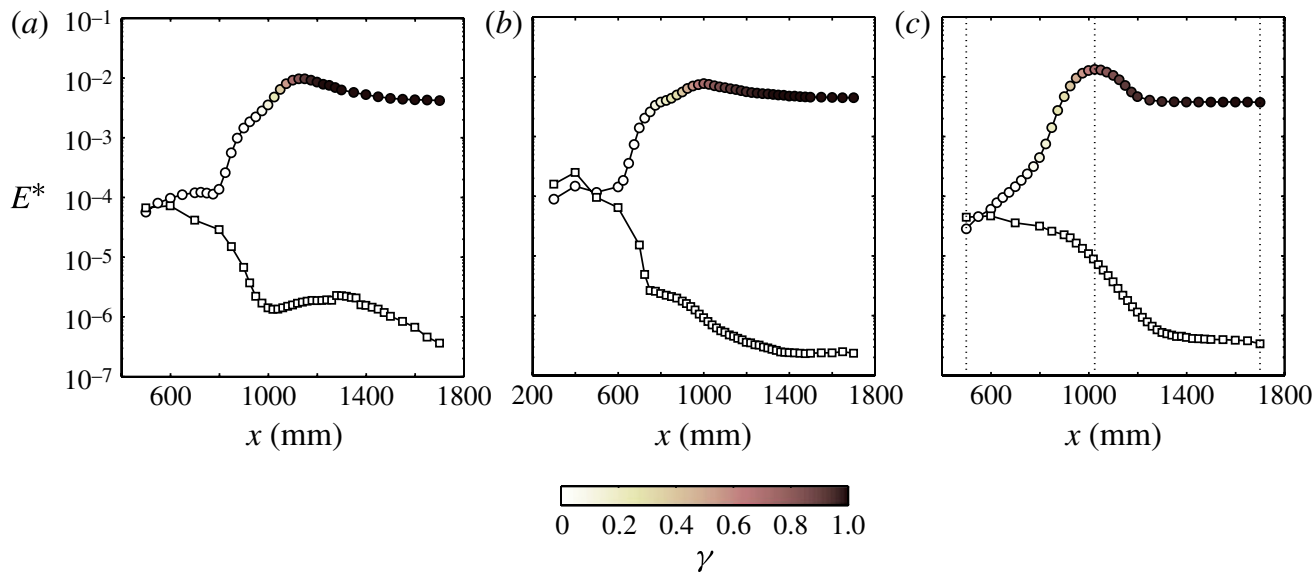

FIGURE 21. (Colour online) Energy $\left(E^{*}\right)$ and intermittency $(\gamma)$ distributions with ( $\square$ ) and without $(\bigcirc)$ MVGs, at different TS forcing frequencies: $(a) F=132$; $(b) F=176$; (c) $F=103$.

On the basis of the present data set, we find the different MVG configurations fairly robust in the sense that a broad range of geometrical MVG parameters works as flow stabilizers.

\subsection{Transition delay by means of miniature vortex generators}

In the previous $\S 5.1$, the attenuation of linear TS waves by means of different MVG configurations was successfully obtained. However, the ability to extrapolate these results to the nonlinear regime and eventually accomplish transition delay is not trivial. In this section, we want to show that the MVGs are, for this set-up, effective in delaying transition to turbulence and hence in reducing skin-friction drag. In figure 21, transition delay is statistically certified by plotting the disturbance energy, $E^{*}=u_{r m s}^{2} / U_{\infty}^{2}$, measured at the wall-normal distance of $y / \delta_{1}=1$ versus the downstream distance for the cases with and without the MVGs. The MVG configuration is $\mathrm{C} 01$ and $(a, b)$ and $(c)$ show the results for three different TS wave forcing frequencies $F=132,176$ and 103, respectively. Prior to the full measurements, the initial forcing amplitude was tuned in each case such that transition was obtained within the measurement region on the flat plate for the two-dimensional reference case. Then the measurements were performed with and without the presence of MVGs. The colour bar shows the intermittency value, $\gamma$, of the velocity signal, with zero corresponding to a purely laminar signal and unity to a fully turbulent signal. It is worth pointing out that the disturbance energy grows and the boundary layer transitions to turbulence in the two-dimensional base flow, but when the MVGs are mounted, the growth is absent and the disturbance energy even decays by two orders of magnitude compared to its initial level at the furthest upstream location.

In figure 22, velocity signals without $(a)$ and with MVGs $(b)$ are shown, corresponding top to bottom to the three successively downstream locations indicated by the vertical dotted lines in figure 21(c). Figure 22 gives a direct comparison between the uncontrolled and passively controlled boundary layer. At the furthest upstream location, the sinusoidal forcing frequency is apparent on the velocity signal in both cases, and in the uncontrolled case it has grown to a really high amplitude, with strong nonlinearities at around $x=1050 \mathrm{~mm}$ from the leading edge; while in 


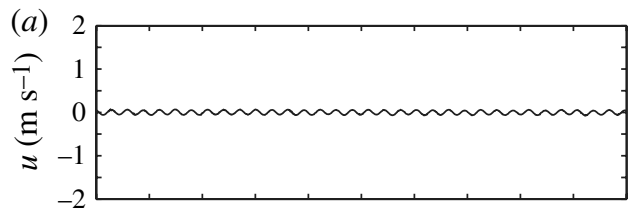

(b)
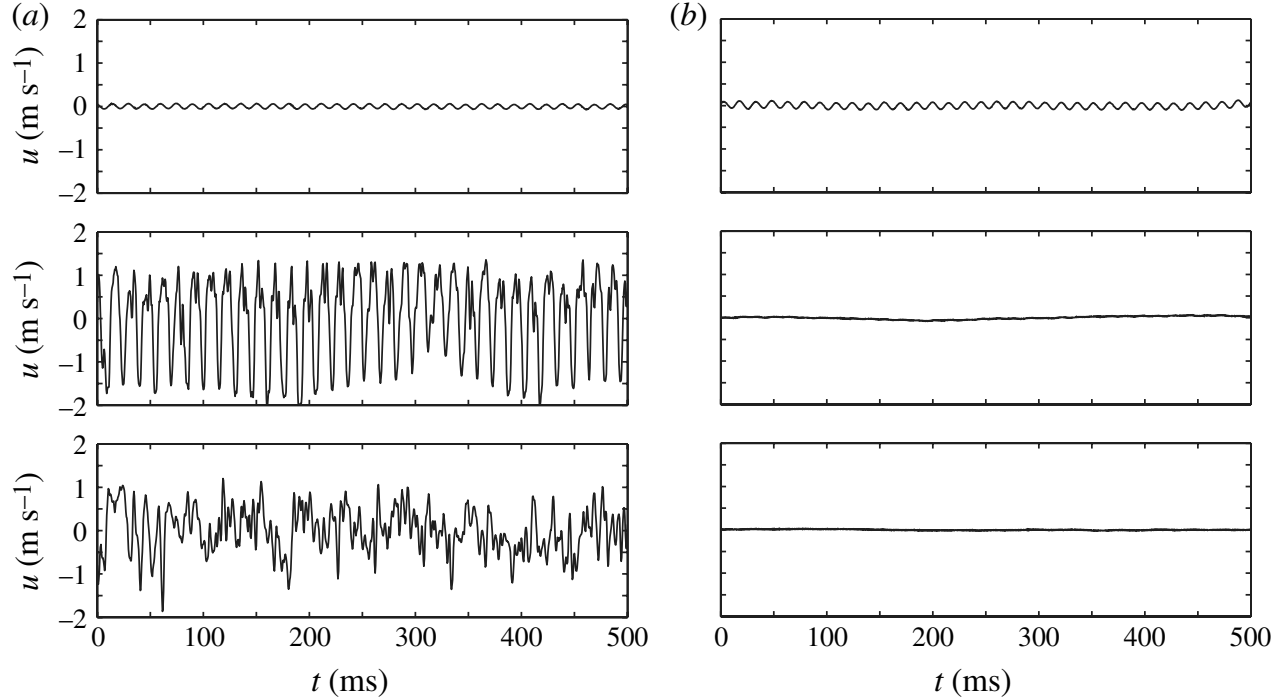

FIGURE 22. Velocity signals for the successively downstream positions indicated by the dotted lines in figure 21(c), (a) without and (b) with MVGs.


FIGURE 23. (Colour online) Energy spectra $\left(e_{f}^{*}\right),(a)$ without and $(b)$ with MVGs, in the downstream direction. The spectra are calculated using the same data as shown in figure 21(c), i.e. at a wall-normal distance above the wall of $y / \delta_{1}=1$.

the controlled case, the initially observed forcing frequency is hardly noticeable at the same downstream location. At the furthest downstream location, the figures display a fully turbulent signal without the MVGs and a purely laminar signal with the MVGs mounted.

The full energy spectra in the frequency-space plane are shown in figure 23 for the data presented in figure $21(c)$. Figure $23(a, b)$ shows the normalized energy spectra $\left(e_{f}^{*}\right)$, which is defined as follows:

$$
e_{f}^{*}=\frac{f \cdot \operatorname{PSD}\{u\}}{u_{r m s}^{2}},
$$


for the uncontrolled and the passively controlled cases, respectively. Here, PSD is the power spectral density, $u$ is the disturbance velocity and $u_{r m s}$ is the standard deviation of $u$. Figure 23(a) shows the full transition scenario with harmonics appearing as a multiple of $n$ of the fundamental frequency mode, i.e. $f=n f_{0}$. Note that $n$ is an integer value equal to or greater than 2 . The energy content of the fundamental mode is at least two orders of magnitude greater than that of any of its harmonics. The figure shows that in addition to the harmonics, there is also a low-frequency mode at $f \simeq 27 \mathrm{~Hz}$ - which, however, dies out fairly quickly. At around $x=1100 \mathrm{~mm}$, the flow has almost completed the transition to turbulence process and a broad energy spectrum has developed. Somewhat further downstream, there is no longer any trace of the initially forced TS frequency. With the MVGs, the energy spectrum in $(b)$ depicts the presence of the low-frequency mode along with the first harmonic (i.e. for $n=2$ ). The fundamental mode is more persistent in the downstream direction with the MVGs compared to the uncontrolled case, but starts to decay, and at $x=1400 \mathrm{~mm}$ it has completely vanished. The low-frequency mode around $27 \mathrm{~Hz}$ seems to be more persistent and extends further downstream compared to the reference case in $(a)$. However, it should be pointed out that this low-frequency mode has a proportionately very low energy content compared to the forced mode with an energy ratio of $\sim 165$. Recall the laminar velocity signal in figure $22(b)$. The source of this low-frequency mode can only be hypothesized upon. Going back to the data adopted in characterizing the experimental set-up, i.e. in the absence of both MVGs and TS waves, this lowfrequency mode is still present. This suggests that it is neither part of the transition scenario nor due to the high-amplitude initial forcing. Possible sources could be an unsteady leading edge stagnation line or trailing edge separation. Independent of the source and the fact that it was present in all experiments we believe that the observed low-frequency and low-energy-content mode has a negligible effect on the reported results.

\section{Summary and conclusions}

In this study, we have explored the possibility of delaying transition to turbulence in a boundary layer flow, and hence reduce skin-friction drag, by means of introducing steady streamwise streaks inside the boundary layer. The streaky base flow is generated passively by using miniature vortex generators (MVGs), which have been shown to be successful in delaying transition caused by two-dimensional waves. Differently from previous investigations, here we set up a fairly realistic flow configuration, i.e. by generating TS waves upstream of the MVG array, allowing the wave to impinge on the array with all its complications, which really challenges the previously reported control strategy.

The boundary layer is carefully characterized, with integral boundary layer parameters calculated both locally and on average over one spanwise wavelength of the streaky base flow. The averaged quantities fall close to the Blasius boundary layer parameters.

Concerning the induced drag due to the presence of the streaks and the extra drag created by the elements themselves, we have been able to show that the skin-friction drag coefficient is increased, with a maximum of $\sim 10 \%$ for most configurations, compared to the Blasius boundary layer. This value can be very small if compared to what can be gained if transition to turbulence is successfully delayed. 
In this paper, a parametric study has been performed by using an integral-based streak amplitude definition, which carries the necessary information on how efficient the streaky base flow is in attenuating the growth of TS waves.

On the basis of the present parametric study, we propose a streak amplitude scaling founded on empiricism. The scaling works satisfactorily considering the available configurations and can be used as a guideline when implementing the passive control, i.e. when mounting the MVGs, in a new flow configuration.

Low-amplitude TS waves have been artificially generated and the effect of the different MVG configurations on the growth curves has been analysed. In order to assure a fair comparison between the streaky base flow cases and the two-dimensional reference base flow, a previously used integral measure of the TS wave amplitude in the cross-sectional plane was applied.

The results show that well-chosen geometrical parameters of the MVG array completely kill the TS wave after some initial growth right behind the MVG array. This initial growth seems to vary with the conventional streak amplitude definition and is believed to be associated with the wall-normal inflectional instability in the low-speed streak, or even in the separated region right behind the MVG blades. If the amplitude reaches the threshold value for bypass transition, the control fails.

The degree of success of the control, as a function of the introduced integral streak amplitude, can be quantified by introducing an attenuation factor. The data show that the optimal streak amplitude for attenuating the growth of TS waves is around $30 \%$ of the free-stream velocity.

Finally, it is shown that the onset of transition may be inhibited altogether throughout the measured region with the presence of an MVG array. The disturbance energy level, based on the full velocity signal, is shown to differ, with close to four orders of magnitude between the reference case and the controlled case.

A comparison between the energy spectra shows that the high initial amplitude forcing decays quickly and does not even allow for harmonics to appear when the MVGs are mounted. This is the opposite of the uncontrolled case, where the full transition scenario may be followed, ending up with a broad energy content over a wide range of frequencies.

Future studies on this topic should address: blade angle variation, which has been kept constant in this study; different types of controlled disturbances; and the possibility of extending the laminar boundary layer even further by mounting a second array downstream of the first one, and in that way being able to regenerate the streaky base flow with a reinforced stabilizing effect, leading to further deferral of the transition onset.

\section{Acknowledgement}

J.H.M.F. acknowledges the European Research Council for its financial support of the AFRODITE project through a Starting Independent Researcher Grant.

\section{REFERENCES}

Andersson, P., Berggren, M. \& Henningson, D. S. 1999 Optimal disturbances and bypass transition in boundary layers. Phys. Fluids 11, 134-150.

Andersson, P., Brandt, L., Bottaro, A. \& Henningson, D. S. 2001 On the breakdown of boundary layer streaks. J. Fluid Mech. 428, 29-60.

Asai, M., Minagawa, M. \& NishiokA, M. 2002 The instability and breakdown of a near-wall low-speed streak. J. Fluid Mech. 455, 289-314. 
Boiko, A. V., Westin, K. J. A., Klingmann, B. G. B., Kozlov, V. V. \& Alfredsson, P. H. 1994 Experiments in a boundary layer subjected to free stream turbulence. Part 2. The role of TS-waves in the transition process. J. Fluid Mech. 281, 219-245.

Butler, K. M. \& FARrell, B. F. 1992 Three-dimensional optimal perturbations in viscous shear flow. Phys. Fluids A 4, 1637-1650.

Camarri, S., Fransson, J. H. M. \& Talamelli, A. 2013 Numerical investigation of the AFRODITE transition control strategy. In Progress in Turbulence $V$ (ed. A. Talamelli, M. Oberlack \& J. Peinke), pp. 65-69. Springer.

Cossu, C. \& BRAndT, L. 2002 Stabilization of Tollmien-Schlichting waves by finite amplitude optimal streaks in the Blasius boundary layer. Phys. Fluids 14, L57-L60.

Cossu, C. \& BRANDT, L. 2004 On Tollmien-Schlichting-like waves in streaky boundary layers. Eur. J. Mech./B Fluids 23, 815-833.

Ellingsen, T. \& Palm, E. 1975 Stability of linear flow. Phys. Fluids 18, 487-488.

Fransson, J. H. M. 2010 Turbulent spot evolution in spatially invariant boundary layers. Phys. Rev. E 81, 035301(R).

Fransson, J. H. M., Brandt, L., Talamelli, A. \& Cossu, C. 2004 Experimental and theoretical investigation of the nonmodal growth of steady streaks in a flat plate boundary layer. Phys. Fluids 16 (10), 3627-3638.

Fransson, J. H. M., Brandt, L., Talamelli, A. \& Cossu, C. 2005 Experimental study of the stabilisation of Tollmien-Schlichting waves by finite amplitude streaks. Phys. Fluids 17, 054110 .

Fransson, J. H. M., Fallenius, B. E. G., Shahinfar, S., Sattarzadeh, S. S. \& TALAMELli, A. 2011 Advanced fluid research on drag reduction in turbulence experiments -AFRODITE-. J. Phys.: Conf. Ser. 318, 032007.

Fransson, J. H. M. \& TAlamelli, A. 2011 Base flow modulations for skin-friction drag reduction. J. Phys.: Conf. Ser. 318, 032008.

Fransson, J. H. M. \& TAlamelli, A. 2012 On the generation of steady streamwise streaks in flat-plate boundary layers. J. Fluid Mech. 698, 211-234.

Fransson, J. H. M., Talamelli, A., Brandt, L. \& Cossu, C. 2006 Delaying transition to turbulence by a passive mechanism. Phys. Rev. Lett. 96, 064501.

Gustavsson, L. H. 1991 Energy growth of three-dimensional disturbances in plane Poiseuille flow. J. Fluid Mech. 224, 241-260.

Hultgren, L. S. \& Gustavsson, L. H. 1981 Algebraic growth of disturbances in a laminar boundary layer. Phys. Fluids 24 (6), 1000-1004.

Johansson, A. V. \& Alfredsson, P. H. 1982 On the structure of turbulent channel flow. J. Fluid Mech. 122, 295-314.

Kachanov, Y. S. 1994 Physical mechanism of laminar boundary-layer transition. Annu. Rev. Fluid Mech. 26, 411-482.

KACHANOV, Y. S. \& TARARYKIN, O. I. 1987 Experimental investigation of a relaxing boundary layer. Proc. Siberian Div. USSR Acad. Sci., Ser. Tech. Sci. 18 (5), 9-19 (in Russian).

KLEBANOFF, P. S. 1971 Effect of free stream turbulence on the laminar boundary layer. Bull. Am. Phys. Soc. 10, 1323.

Klingmann, B. G. B., Boiko, A., Westin, K. J. A., Kozlov, V. V. \& Alfredsson, P. H. 1993 Experiments on the stability of Tollmien-Schlichting waves. Eur. J. Mech.B/Fluids 12, 493-514.

Kurian, T. \& Fransson, J. H. M. 2011 Transient growth in the asymptotic suction boundary layer. Exp. Fluids 51, 771-784.

LANDAhL, M. T. 1980 A note on an algebraic instability of inviscid parallel shear flows. J. Fluid Mech. 98, 243-251.

Lindgren, B. \& Johansson, A. V. 2002 Design and evaluation of a low-speed wind-tunnel with expanding corners. TRITA-MEK Tech. Rep. 2002:14. KTH, Stockholm.

LiU, Y., ZAKI, T. A. \& DURBin, P. A. 2008 Boundary-layer transition by interaction of discrete and continuous modes. J. Fluid Mech. 604, 199-233.

Lögdberg, O., Fransson, J. H. M. \& Alfredsson, P. H. 2009 Streamwise evolution of longitudinal vortices in a turbulent boundary layer. J. Fluid Mech. 623, 27-58. 
LUCHINI, P. 1996 Reynolds-number independent instability of the boundary layer over a flat surface. J. Fluid Mech. 327, 101-115.

LUCHINI, P. 2000 Reynolds-number independent instability of the boundary layer over a flat surface. Part 2: optimal perturbations. J. Fluid Mech. 404, 289-309.

Morkovin, M. V. 1969 The many faces of transition. In Viscous Drag Reduction (ed. C. S. Wells), Plenum Press.

Reddy, S. C. \& Henningson, D. S. 1993 Energy growth in viscous channel flows. J. Fluid Mech. 252, 209-238.

Reshotko, E. 2001 Transient growth: a factor in bypass transition. Phys. Fluids 13, 1067-1075.

Schlatter, P., Deusebio, E., De LAnge, R. \& BRAndt, L. 2010 Numerical study of the stabilisation of boundary-layer disturbances by finite amplitude streaks. Intl J. Flow Control 2, 259-288.

SCHLichting, H. 1933 Berechnung der anfachung kleiner störungen bei der plattenströmung. Z. Angew. Math. Mech. 13, 171-174.

Schmid, P. J. \& Henningson, D. S. 2001 Stability and Transition in Shear Flows. Springer.

Schubauer, G. B. \& Skramstad, H. K. 1947 Laminar boundary layer oscillations and the stability of laminar flow. J. Aero. Sci. 14, 69-78.

Shahinfar, S., Sattarzadeh, S. S., Fransson, J. H. M. \& Talamelli, A. 2012 Revival of classical vortex generators now for transition delay. Phys. Rev. Lett. 109, 074501.

Tollmien, W. 1929 Über die entstehung der turbulenz. Nachr. Ges. Wiss. Göttingen 21-24 (English translation NACA TM 609, 1931).

Trefethen, L. N., Trefethen, A. E., Reddy, S. C. \& Driscoll, T. A. 1993 Hydrodynamic stability without eigenvalues. Science 261, 578-584.

White, E. B. 2002 Transient growth of stationary disturbances in a flat plate boundary layer. Phys. Fluids 14, 4429-4439.

White, E. B., Rice, J. M. \& ERgin, G. 2005 Receptivity of stationary transient disturbances to surface roughness. Phys. Fluids 17, 064109. 